\title{
Radiation From Flux Flow In Josephson Junction Structures
}

\author{
L.N. Bulaevskii ${ }^{1}$ and A.E. Koshelev ${ }^{2}$ \\ ${ }^{1}$ Los Alamos National Laboratory, Los Alamos, New Mexico 87545 \\ ${ }^{2}$ Materials Science Division, Argonne National Laboratory, Argonne, Illinois 60439
}

(Dated: April 14, 2021)

\begin{abstract}
We derive the radiation power from a single Josephson junction (JJ) and from layered superconductors in the flux-flow regime. For the JJ case we formulate the boundary conditions for the electric and magnetic fields at the edges of the superconducting leads using the Maxwell equations in the dielectric media and find dynamic boundary conditions for the phase difference in JJ which account for the radiation. We derive the fraction of the power fed into JJ transformed into the radiation. In a finite-length JJ this fraction is determined by the dissipation inside $\mathrm{JJ}$ and it tends to unity as dissipation vanishes independently of mismatch of the junction and dielectric media impedances. We formulate also the dynamic boundary conditions for the phase difference in intrinsic Josephson junctions in highly anisotropic layered superconductors of the $\mathrm{Bi}_{2} \mathrm{Sr}_{2} \mathrm{CaCu}_{2} \mathrm{O}_{8}$ type at the boundary with free space. Using these boundary conditions, we solve equations for the phase difference in the linear regime of Josephson oscillations for rectangular and triangular lattices of Josephson vortices. In the case of rectangular lattice for crystals with the thickness along the $c$-axis much larger than the radiation wavelength we estimate the radiation power per unit length in the direction of magnetic field at the frequency $1 \mathrm{THz}$ as $\sim N \mu \mathrm{W} / \mathrm{cm}$ for $\mathrm{Tl}_{2} \mathrm{Ba}_{2} \mathrm{CaCu}_{2} \mathrm{O}_{8}$ and $\sim 0.04 N$ $\mu \mathrm{W} / \mathrm{cm}$ for $\mathrm{Bi}_{2} \mathrm{Sr}_{2} \mathrm{CaCu}_{2} \mathrm{O}_{8}$. For crystals with thickness smaller than the radiation wavelength we found that the radiation power in the resonance is independent on number of layers and can be estimated at $1 \mathrm{THz}$ as $0.5 \mathrm{~W} / \mathrm{cm}\left(\mathrm{Tl}_{2} \mathrm{Ba}_{2} \mathrm{CaCu}_{2} \mathrm{O}_{8}\right)$ and $24 \mathrm{~mW} / \mathrm{cm}\left(\mathrm{Bi}_{2} \mathrm{Sr}_{2} \mathrm{CaCu}_{2} \mathrm{O}_{8}\right)$. For rectangular lattice, due to superradiation regime, up to half of power fed into the crystal may be converted into the radiation. In the case of triangular or random lattice in the direction perpendicular to the layers the fraction of power converted into the radiation depends on the dissipation rate and is much lower than for rectangular lattice in the case of high-temperature superconductors with nodes in the gap.
\end{abstract}




\section{INTRODUCTION}

In 1962 Josephson [1] predicted electromagnetic radiation from superconducting tunneling junction arguing that in the presence of voltage $V$ across the junction the phase difference $\varphi$ changes with time as $\partial \varphi / \partial t=2 e V / \hbar$, while the tunneling current density $J$ changes as $J=J_{c} \sin (2 \mathrm{eVt} / \hbar)$. Here $J_{c}$ is the critical superconducting current via the junction. Thus the photons with the Josephson frequency $\omega=2 \mathrm{eV} / \hbar$ may be emitted from Josephson junction (JJ). Such a radiation from JJ into the waveguide with the power $\mathcal{P}_{\text {rad }} \sim 10^{-12} \mathrm{~W}$ has been detected by Dmitrenko et al. [2] and by Langenberg et al. [3] soon after the Josephson prediction. The junction in both experiments was subject to bias current and dc magnetic field applied parallel to the junction. The dc magnetic field induces Josephson vortices and in the presence of the transport current across the junction they move to the JJ edge causing oscillations of the magnetic and electric fields inside JJ and in superconducting leads around JJ. More precisely, flux flow induces electromagnetic (Swihart) waves inside JJ, and they are partially transmitted outside when hit JJ edges [4]. From measurements of the current-voltage $(I-V)$ characteristics, Langenberg et al. [3] found the power $\mathcal{P}=I V$ fed into the junction and the fraction converted into radiation, $Q=\mathcal{P}_{\text {rad }} / \mathcal{P} \approx 10^{-5}$. Theoretically $Q$ was estimated as a ratio of the impedances of $\mathrm{JJ}$ modeled as a strip line and the waveguide [3,, 5$]$ :

$$
Q_{Z}=\frac{4 Z_{0} Z_{s}}{\left(Z_{0}+Z_{s}\right)^{2}} \sim\left(\frac{8 \lambda d}{\varepsilon_{i} w^{2}}\right)^{1 / 2}
$$

where $Z_{0}$ is the waveguide impedance, $Z_{s}$ is the strip line impedance in the limit $k_{\omega} w \ll 1$ and $Z_{s} \ll Z_{0}$. Here $\lambda$ is the London penetration length of the superconducting leads, $d$ is the thickness of the insulating layer, $\varepsilon_{i}$ is its dielectric constant, $k_{\omega}=\omega / c$ and $w$ is the junction width. Eq. (11) gives $\approx 10^{-5}$ for the studied junction in agreement with experimental value. Thus low radiation power from $\mathrm{JJ}$ was attributed to the mismatch between the impedances of the junction and the waveguide. To the best of our knowledge, no deeper theoretical treatment of radiation was made after that and the concept of the impedance mismatch for a single $\mathrm{JJ}$ and later for layered superconductors with intrinsic JJ was accepted by the community.

As radiation from the vortex flow in a single junction was found to be quite low, a natural idea was proposed to use multiple lock-in junctions. Then, in the super-radiation regime, radiation power may be enhanced by the factor $N^{2}$, where $N$ is the number of synchronized junctions inside the space of the radiation wavelength. Intensive theoretical and experimental study was done in 
this direction, see e.g. Ref. 6, 7, 8, 9, 10, 11, 12. However, an effective way to synchronize many junctions was not found so far. There are two problems to overcome for synchronization. The first one is the technologically inevitable variation of parameters from junction to junction, mainly $J_{c}$ parameter, which affects operating frequency of the junction at given current. The second problem is that the coherent locked-in flow of the Josephson vortices in different junctions is unstable in a wide range of parameters.

The discovery of layered high-temperature superconductivity added new breath into this activity. It was recognized that the $\mathrm{Bi}$ - and Tl-based cuprate superconductors with weakly coupled superconducting $\mathrm{CuO}_{2}$ layers exhibit the same static and dynamical Josephson properties as artificial tunneling junctions. In another words, the crystals $\mathrm{Bi}_{2} \mathrm{Sr}_{2} \mathrm{CaCu}_{2} \mathrm{O}_{8}(\mathrm{BSCCO})$ and $\mathrm{Tl}_{2} \mathrm{Ba}_{2} \mathrm{CaCu}_{2} \mathrm{O}_{8}$ (TBCCO) represent a stack of many intrinsic Josephson junctions on the atomic scale [13]. The first indication of the intrinsic Josephson effect was observation of switching of individual junctions to the resistive state in the $I-V$ characteristics [14]. After that many Josephson effects have been found in these systems including the Fraunhofer patterns in the dependence of the critical current on the dc magnetic field applied parallel to the layers [15], Josephson plasma resonance [16] strongly affected by pancake vortices in the presence of the magnetic field applied perpendicular to the layers [17, 18], Shapiro steps in the $I-V$ characteristics induced by external microwave radiation [19, 20], and Fiske resonances [21, 22, 23]

One can anticipate much smaller variations of intrinsic-junction parameters in comparison with the artificially-fabricated junction arrays. In addition, the intrinsic junctions are much closer to each other and one can anticipate much stronger coupling between them. What is more, there are many junctions on the scale of the radiation wavelength, and so they will super-radiate when synchronized. Due to these advantages, the moving Josephson vortex lattice in layered superconductors was proposed as a source of monochromatic tunable continuous electromagnetic radiation in the terahertz frequency range [24, 25, 26, 27, 28]. Experimentally, radiation from the high-temperature layered superconductor BSCCO at relatively low frequencies, 7-16 GHz, was detected by Hechtfischer et al. [29]. Some indirect evidence of radiation at higher frequencies has been recently reported by Kadowaki et al. [30] and Batov et al. [31] reported radiation at the frequency $0.5 \mathrm{THz}$ with power $1 \mathrm{pW}$ from BSCCO mesa consisting 100 junctions in zero dc magnetic field.

Therefore, it is interesting to estimate theoretically the possible radiation power generated by a moving vortex lattice and find optimal conditions for generation. For that we need to understand 
the mechanism of conversion of the electromagnetic field associated with the flux flow of the Josephson vortices into the electromagnetic waves outside of the Josephson junction (JJ) and find limitations imposed by this mechanism in Josephson tunneling structures. A natural first step is to understand at the microscopic level the conversion mechanism in a single JJ. Then such an approach may be extended to layered structures.

In the first part we consider radiation from a single JJ. Then the method to treat radiation is extended to intrinsic junctions in layered superconductors. We will show that super-radiation regime is inherent to moving rectangular vortex lattice in such crystals. We will discuss the consequences of this regime for the radiation power and $I-V$ characteristics.

\section{RADIATION FROM A SINGLE JOSEPHSON JUNCTION}

As we discussed in the Introduction, a common way to evaluate the radiation out of a single Josephson junction is to take product of the total power supplied to the junction, $\mathcal{P}=I V$ and the impedance mismatch coefficient $Q_{Z}$, Eq. (11). A weak point of this impedance approach for real, finite-length $\mathrm{JJ}$ is ignoring of multiple reflections inside the junction, i.e., assumption that the propagating electromagnetic wave has only one attempt to escape the JJ, decaying before reflected wave reaches another edge. In fact, at low dissipation rate (low temperature and low dielectric losses inside the insulating layer) reflections lead to the formation of almost standing Swihart waves inside JJ. In this case $Q$ strongly depends on the dissipation and approaches unity as dissipation vanishes. Then the question turns out to be what are limitations on $\mathcal{P}$ and $\mathcal{P}_{\text {rad }}$ rather than on $Q$.

On the other hand, the standard analysis of transport properties of finite-size JJs uses sineGordon equation and zero-derivative boundary conditions for the oscillating part of the phase at the edges [5, 32, 33, 34]. At the JJ edges vanishing oscillating magnetic field $B=\partial \varphi / \partial x$ leads to zero Poynting vector. Thus for such boundary conditions the outside radiation is absent and all supplied power dissipates inside JJ.

In the following we reconsider this problem, discuss the power conversion mechanism and derive the radiation power from a single $\mathrm{JJ}$ into free space in the case when $w$ is much larger than the wavelength of outcoming electromagnetic wave. This is not realistic limit, but it is a simplest one which demonstrates the method to treat radiation and which may be directly extended to the case of smaller $w$. Our rigorous approach is based on solution of the Maxwell equations 


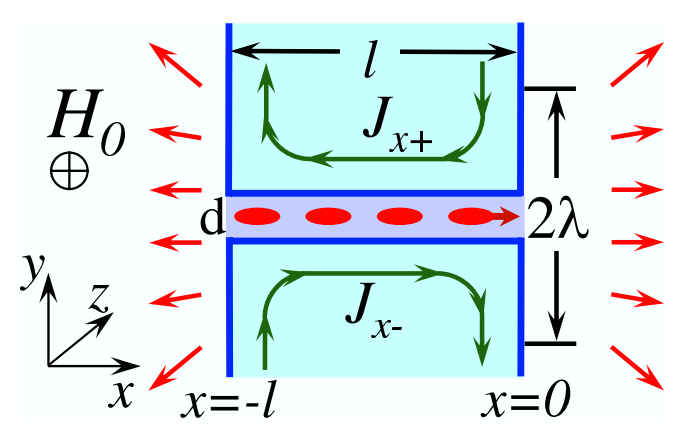

FIG. 1: A finite-size Josephson junction opened into free space at both edges. Ellipses illustrate the moving vortex lattice. Curved lines show the screening currents inside the superconducting leads, arrows show radiation from area $2 \lambda$ around the junction. The applied dc magnetic field $H_{0}$ is along the $z$-axis.

inside the superconducting leads and in outside space, which allowed us to formulate accurate dynamic boundary conditions for the oscillating phase inside JJ. In the linear regime of Josephson oscillations we obtained analytical results for $\mathcal{P}_{\text {rad }}$ and $Q$ using the perturbation theory. In this regime we found that $Q \propto Q_{Z} \mathcal{N}$, where $\mathcal{N}$ is the number of reflections before Swihart wave decays inside JJ. At low temperatures in JJ made out of gapped superconductors with perfect insulating layer $\mathcal{N}$ may be large compensating small $Q_{Z}$. Our approach also opens the way for numerical calculations in general case, when linear approach is invalid.

The Josephson junctions with low level of the dissipation become available now due to the perspective to use them as a qubits for quantum computing [35]. The radiation should be stronger in such junctions in comparison with previously studied ones. This gives additional motivation to reconsider the theoretical background of the radiation from JJ.

To find outside radiation due to the Josephson oscillations, one has to match oscillating fields inside the junction and in the superconducting leads with the wave solution outside the junction. Expressing the oscillating fields inside the junctions via the phase difference, we derive dynamic boundary conditions for the phase difference and relate the Poynting vector of radiation to the phase difference at junction edge. The final step is the solution of the equations for the phase difference accounting for the dynamic boundary conditions and derivation of the Poynting vector.

\section{Equation for the phase difference}

We consider a JJ with the length $l \gg \lambda$ located at $-l<x<0$ and bounded by dielectric with dielectric constant $\varepsilon_{d}$, see Fig. 1 Strength of the coupling in the junction is char- 
acterized by the Josephson current density $J_{c}$ and related parameters: the Josephson length, $\lambda_{J}=\sqrt{c \Phi_{0} /\left(8 \pi^{2} \Lambda J_{c}\right)}$, and plasma frequency, $\omega_{p}=\sqrt{8 \pi^{2} d c J_{c} /\left(\varepsilon_{i} \Phi_{0}\right)}$, where $\Lambda=2 \lambda+d$. We consider the simplest situation when the JJ width along $z$ direction, $w$, is larger than both $\lambda_{J}$ and wavelength of outcoming electromagnetic wave. We will consider straight Josephson vortices along the external magnetic field. In this approach the problem becomes one-dimensional for the phase difference and two-dimensional for the electric and magnetic fields in the outer space as both of them become $z$-independent. We consider junction in resistive state and assume that the junction phase, $\bar{\varphi}$, oscillates with the Josephson frequency $\omega$ generating oscillating electric $\left(E_{x}\right.$ and $\left.E_{y}\right)$ and magnetic $\left(B_{z}\right)$ fields inside the junction and in the superconducting leads. Our task is to find spatial distribution of these fields and match them with outside fields to find equation and boundary conditions for $\tilde{\varphi}$.

We first derive equation for the oscillating magnetic field inside the superconducting leads. We use complex representation for the oscillating fields and phase, e.g.,

$$
\bar{\varphi}(x, t)=\langle\bar{\varphi}(x, t)\rangle_{t}+\sum_{\omega} \operatorname{Re}\left[\bar{\varphi}_{\omega}(x) e^{-i \omega t}\right]
$$

Here $\langle\ldots\rangle_{t}$ means time average. The phase gradient is connected by the following relation with the magnetic field inside the junction and supercurrents flowing along the junction at the opposite sides

$$
\nabla_{x} \bar{\varphi}=\frac{8 \pi^{2} \lambda^{2}}{c \Phi_{0}}\left[J_{x+}-J_{x-}\right]-\frac{2 \pi d}{\Phi_{0}} B_{z} .
$$

From Maxwell equations, material equation for current inside superconductor, $\mathbf{J}=$ $\left(c / 4 \pi \lambda^{2}\right)\left[\left(\Phi_{0} / 2 \pi\right) \nabla \phi-\mathbf{A}\right]+\sigma_{q} \mathbf{E}$, London relation for the electric field, $\mathbf{E}=-\left(4 \pi \lambda^{2} i \omega / c\right) \mathbf{J}$, and Eq. (2), we derive the following equation for the oscillating magnetic field inside the leads $(-l<x<0)$ at $d \ll \lambda$

$$
\begin{aligned}
& \left(\nabla_{x}^{2}+\nabla_{y}^{2}\right) B_{z}(\omega)-\frac{B_{z}(\omega)}{\lambda_{\omega}^{2}}=\frac{\Phi_{0}}{2 \pi \lambda^{2}} \frac{\partial \bar{\varphi}_{\omega}}{\partial x} \delta(y), \\
& \lambda_{\omega}^{-2}=\lambda^{-2}-\varepsilon_{s} k_{\omega}^{2}+4 \pi i k_{\omega} \sigma_{q} / c, \quad k_{\omega}=\omega / c .
\end{aligned}
$$

We ignore small contribution to the magnetic field from the dc current flowing via the junction. The ac electric field inside the superconducting leads is $E_{y}(\omega, x, y)=i \lambda_{\omega}^{2} k_{\omega} \nabla_{x} B_{z}(\omega, x, y)$. To obtain the total electric field in the junction area, one has to account also for the field inside the dielectric layer. This gives

$$
E_{y}(\omega, x, y)=-\frac{i \omega \Phi_{0}}{2 \pi c} \bar{\varphi}_{\omega}(x) \delta(y)+i \lambda_{\omega}^{2} k_{\omega} \nabla_{x} B_{z}(\omega, x, y)
$$


The boundary condition for $\bar{\varphi}_{\omega}(x)$ follows from the boundary condition for the electric field

$$
-i \omega\left[\varepsilon_{d} E_{x}(+0, y)-\varepsilon E_{x}(-0, y)\right]=4 \pi J_{x}(-0, y) .
$$

As $E_{x}(-0, y)=-i \omega\left(4 \pi \lambda^{2} / c^{2}\right) J_{x}(-0, y)$, we obtain

$$
4 \pi\left(1-\varepsilon k_{0}^{2} \lambda^{2}\right) J_{x}(-0, y)=-i \omega \varepsilon_{d} E_{x}(+0, y)
$$

As the electric field $E_{x}(+0, y)$ is continuous at $y=0$, this means that $J_{x}(0, y)$ also must be continuous and from Eq. (2) in the limit $d \ll \lambda$ we obtain

$$
\nabla_{x} \bar{\varphi}_{\omega}(0)=\nabla_{x} \bar{\varphi}_{\omega}(-l)=0 .
$$

We can represent solution of Eq. (3) near the edge $x=0$ as

$$
\begin{aligned}
& B_{z}(x, y)=B_{b}(x, y)-\frac{\Phi_{0}}{(2 \pi \lambda)^{2}} \int_{-\infty}^{0} \nabla_{x^{\prime}} \bar{\varphi}_{\omega}\left(x^{\prime}\right) d x^{\prime} \times \\
& {\left[K_{0}\left(c_{-}\right)-K_{0}\left(c_{+}\right)\right], \quad c_{ \pm}=\sqrt{\left(x \pm x^{\prime}\right)^{2}+y^{2}} / \lambda_{\omega}}
\end{aligned}
$$

where $K_{0}(z)$ is the modified Bessel function and $B_{b}(x, y)$ is the solution of the homogeneous equation

$$
\left(\nabla_{x}^{2}+\nabla_{y}^{2}\right) B_{b}-\lambda_{\omega}^{-2} B_{b}=0
$$

with the boundary condition $B_{b}(-0, y)=B_{z}(+0, y)$. As a function of $x, B_{b}(x, y)$ decays at distance $\sim \lambda$ from the boundary. For its Fourier transform along the $y$ direction, we obtain $\left[\kappa_{x}=\right.$ $\left.\left(\lambda_{\omega}^{-2}+k_{y}^{2}\right)^{1 / 2}\right]$

$$
B_{b}\left(\omega, x, k_{y}\right)=B_{z}\left(+0, k_{y}\right) \exp \left(-\kappa_{x}|x|\right)
$$

For static case, distribution of the magnetic field near the edge of JJ has been recently derived by A. Gurevich (unpublished).

Using Maxwell equation $\nabla_{x} B_{z}=\left(i \omega \varepsilon_{i} / c\right) E_{y}-(4 \pi / c) J_{y}$, Josephson relation $E_{y}(x, 0)=$ $-i \omega \Phi_{0} \bar{\varphi}_{\omega}(x) /(2 \pi c d)$, and Eq. (8), we find equation for the oscillating phase

$$
\begin{aligned}
& \left(\frac{\omega^{2}}{\omega_{p}^{2}}+\alpha_{t} \frac{i \omega}{\omega_{p}}\right) \bar{\varphi}_{\omega}(x)+\frac{\lambda_{J}^{2}}{\pi \lambda} \int_{-\infty}^{0} d x^{\prime} \nabla_{x}\left[K_{0}\left(\frac{x-x^{\prime}}{\lambda_{\omega}}\right)+\right. \\
& \left.K_{0}\left(\frac{x+x^{\prime}}{\lambda_{\omega}}\right)\right] \nabla_{x^{\prime}} \bar{\varphi}_{\omega}\left(x^{\prime}\right)-\frac{c \nabla_{x} B_{b}(x, 0)}{4 \pi J_{c}}=s_{\omega}(x),
\end{aligned}
$$

where $s_{\omega}(x)$ is the complex amplitude of the oscillating Josephson current, $\sin [\bar{\varphi}(x, t)]=$ $\operatorname{Re}\left[s_{\omega}(x) \exp (-i \omega t)\right]$, and $\alpha_{t}=\omega_{p} \sigma_{t} \Phi_{0} /\left(2 \pi J_{c} c d\right)$ is damping due to the tunneling quasiparticle 
conductivity. Similar nonlocal equation has been derived by Gurevich [36]. In the case $\lambda \ll \lambda_{J}$ and $|x| \gg \lambda$ non-locality is not essential and Eq. (10) can be reduced to usual local approximate equation

$$
\left(\frac{\omega^{2}}{\omega_{p}^{2}}+\alpha_{t} \frac{i \omega}{\omega_{p}}\right) \varphi_{\omega}+\lambda_{J}^{2}\left(1-\alpha_{q} \frac{i \omega}{\omega_{p}}\right) \nabla_{x}^{2} \varphi_{\omega}=s_{\omega}(x),
$$

where $\alpha_{q}=2 \pi \lambda^{2} \omega_{p} \sigma_{q} / c^{2}$ is the dissipation due to quasiparticles inside superconductor and we used expansion $\lambda_{\omega} / \lambda \approx 1-2 \pi i \lambda^{2} k_{\omega} \sigma_{q} / c$ neglecting $k_{\omega}^{2}$ term.

\section{Boundary conditions and the Poynting vector}

Near the boundary situation is more complicated because, in addition to smoothly changing part, the phase has component decaying at distances of order $\lambda$ from the boundary. This extra phase is smaller than the smooth phase by the factor $\sim \lambda / \lambda_{J}$ but it has comparable derivative. Our purpose is to derive accurate boundary condition for the smooth phase, $\varphi_{\omega}(x)$, obeying Eq. (11). For this we integrate Eq. (10) from intermediate distance $-x_{i}$ with $\lambda \ll x_{i} \ll \lambda_{J}$ up to the boundary $x=0$. Neglecting small terms proportional to $x_{i}$ and $B_{b}\left(-x_{i}, 0\right)$ [for $B_{z}(0, y) \ll$ $\left.\Phi_{0} /\left(4 \pi \lambda^{2}\right)\right]$, using local approximation at $x=-x_{i}$, we obtain the boundary condition for the smooth phase in a very simple form

$$
\nabla_{x} \varphi_{\omega}(0) \approx \nabla_{x} \bar{\varphi}_{\omega}\left(-x_{i}\right)=-\frac{\lambda}{\lambda_{\omega}} \frac{4 \pi \lambda}{\Phi_{0}} B_{z}(\omega, \mathbf{r}=0)
$$

This equation can be compared with condition (7) for the total phase. Eq. (12) allows us reduce the problem to solution of local equation (11) for smooth phase with modified boundary conditions and avoid solving exact integral equation for the total phase, Eqs. (7) and (10).

For that we need to express $B_{z}(\mathbf{r}=0)$ via $\varphi_{\omega}$. Relation between the electric and magnetic field at the boundary is determined by properties of outside media.

\section{Outside dielectric media}

We consider first outside dielectric media with dielectric constant $\varepsilon_{d}$. In the situation $w k_{\omega} \gg 1$, in the straight-vortices approach, outside fields are $z$-independent. In addition, if we assume that the dielectric media is infinite in $y$ direction and the thickness of the leads is much larger than $\lambda$, we can use Fourier transform in this direction. In this case the Fourier components of fields with 
$\left|k_{y}\right|<\sqrt{\varepsilon_{d}} k_{\omega}$ propagate in the media while the field components with $\left|k_{y}\right|>\sqrt{\varepsilon_{d}} k_{\omega}$ decay. In particular, for $E_{y}\left(\omega, x, k_{y}\right)$ we have

$$
E_{y}\left(\omega, x, k_{y}\right)=\left\{\begin{array}{c}
E_{y}\left(\omega, 0, k_{y}\right) \exp \left[i \sqrt{\varepsilon_{d} k_{\omega}^{2}-k_{y}^{2}} \operatorname{sign}(\omega) x\right], \quad \text { for }\left|k_{y}\right|<\sqrt{\varepsilon_{d}} k_{\omega}, \\
E_{y}\left(\omega, 0, k_{y}\right) \exp \left[-\sqrt{k_{y}^{2}-\varepsilon_{d} k_{\omega}^{2}} x\right], \quad \text { for }\left|k_{y}\right|>\sqrt{\varepsilon_{d}}\left|k_{\omega}\right| .
\end{array}\right.
$$

Other field components, $E_{x}$ and $B_{z}$, can be expressed via $E_{y}\left(\omega, 0, k_{y}\right)$. First, $E_{x}\left(\omega, x, k_{y}\right)$ can be obtained from Eq. (13) and Maxwell equation $\nabla \mathbf{E}=0$ and then $B_{z}\left(\omega, x, k_{y}\right)$ can be obtained using the Maxwell equation $(\nabla \times \mathbf{E})_{z}=i k_{\omega} B_{z}$ which gives

$$
\begin{aligned}
& B_{z}\left(\omega, x, k_{y}\right)=\frac{\varepsilon_{d}\left|k_{\omega}\right|}{\sqrt{\varepsilon_{d} k_{\omega}^{2}-k_{y}^{2}}} E_{y}\left(\omega, 0, k_{y}\right) \exp \left[i \sqrt{\varepsilon_{d} k_{\omega}^{2}-k_{y}^{2}} \operatorname{sign}(\omega) x\right], \quad \text { for }\left|k_{y}\right|<\sqrt{\varepsilon_{d}} k_{\omega}, \\
& B_{z}\left(\omega, x, k_{y}\right)=\frac{-i \varepsilon_{d} k_{\omega}}{\sqrt{k_{y}^{2}-\varepsilon_{d} k_{\omega}^{2}}} E_{y}\left(\omega, 0, k_{y}\right) \exp \left[-\sqrt{k_{y}^{2}-\varepsilon_{d} k_{\omega}^{2}} x\right], \quad \text { for }\left|k_{y}\right|>\sqrt{\varepsilon_{d}} k_{\omega}
\end{aligned}
$$

In particular, this gives relation between fields at the boundary, which we will use to formulate the boundary conditions for the phase

$$
\begin{aligned}
& B_{z}\left(0, k_{y}\right)=\zeta\left(\omega, k_{y}\right) E_{y}\left(0, k_{y}\right), \\
& \zeta\left(\omega, k_{y}\right)=\left\{\begin{array}{c}
\left|k_{\omega}\right| \varepsilon_{d} / \sqrt{\varepsilon_{d} k_{\omega}^{2}-k_{y}^{2}}, \text { for }\left|k_{y}\right|<\sqrt{\varepsilon_{d}}\left|k_{\omega}\right|, \\
-i k_{\omega} \varepsilon_{d} / \sqrt{k_{y}^{2}-\varepsilon_{d} k_{\omega}^{2}}, \text { for }\left|k_{y}\right|>\sqrt{\varepsilon_{d}}\left|k_{\omega}\right| .
\end{array}\right.
\end{aligned}
$$

Note again that the term $\zeta\left(\omega, k_{y}\right)$ for $\left|k_{y}\right|<\sqrt{\varepsilon_{d}} k_{\omega}$ originates from outcoming electromagnetic wave (radiation), while the term $\zeta\left(\omega, k_{y}\right)$ for $\left|k_{y}\right|>\sqrt{\varepsilon_{d}} k_{\omega}$ is due to the wave decaying at distance $\sim\left(k_{y}^{2}-\varepsilon_{d} k_{\omega}^{2}\right)^{-1 / 2}$ from the lead boundaries. The latter term does not carry energy out of the junction. For completeness, we also present this important relation in the frequency-space representation

$$
\begin{aligned}
B_{z}(\omega, 0, y) & =\int d y^{\prime} U\left(\omega, y-y^{\prime}\right) E_{y}\left(\omega, 0, y^{\prime}\right) \\
U(\omega, y) & =\frac{\varepsilon_{d}}{2}\left[\left|k_{\omega}\right| J_{0}\left(\sqrt{\varepsilon_{d}} k_{\omega} y\right)+i k_{\omega} N_{0}\left(\sqrt{\varepsilon_{d}} k_{\omega} y\right)\right] .
\end{aligned}
$$

where $J_{0}(z)$ and $N_{0}(z)$ are the Bessel functions, and in the time-space representation

$$
B_{z}(t, 0, y)=\sqrt{\varepsilon_{d}} \frac{\partial}{\partial t} \int d t^{\prime} d y^{\prime} \frac{\Theta\left[c_{d}^{2}\left(t-t^{\prime}\right)^{2}-\left(y-y^{\prime}\right)^{2}\right]}{\sqrt{c_{d}^{2}\left(t-t^{\prime}\right)^{2}-\left(y-y^{\prime}\right)^{2}}} E_{y}\left(t^{\prime}, 0, y^{\prime}\right)
$$

where $\Theta(x)=1$ if $x>0$ and 0 if $x<0$ and $c_{d}=c / \sqrt{\varepsilon_{d}}$.

Now we will relate boundary fields with the phase. From Eqs. (5) and (8) follows the relation

$$
\begin{aligned}
& E_{y}\left(0, k_{y}\right)=i \lambda_{\omega}^{2} k_{\omega} \kappa_{x} B_{z}\left(0, k_{y}\right)- \\
& \frac{i k_{\omega} \Phi_{0}}{2 \pi}\left(\varphi_{\omega}(0)-\frac{\lambda_{\omega}^{2}}{\lambda^{2}} \int_{-\infty}^{0} \exp \left(\kappa_{x} x\right) \nabla_{x} \varphi_{\omega}(x) d x\right) .
\end{aligned}
$$


This relation and Eq. (15) allow us to express the boundary fields via the phase distribution

$$
\begin{aligned}
& E_{y}\left(0, k_{y}\right)=\frac{B_{z}\left(0, k_{y}\right)}{\zeta\left(\omega, k_{y}\right)}, \quad B_{z}\left(0, k_{y}\right)=\frac{\Phi_{0}}{2 \pi} \frac{i \zeta k_{\omega}}{1-i \zeta \lambda_{\omega}^{2} k_{\omega} \kappa_{x}} \times \\
& \left(-\varphi_{\omega}(0)+\frac{\lambda_{\omega}^{2}}{\lambda^{2}} \int_{-\infty}^{0} \exp \left(\kappa_{x} x\right) \nabla_{x} \varphi_{\omega}(x) d x\right) .
\end{aligned}
$$

Typically, $\zeta \lambda_{\omega}^{2} k_{\omega} \kappa_{x} \sim \lambda \omega / c \ll 1$. Also $\nabla_{x} \varphi_{\omega}(x) \sim \varphi_{\omega}(x) / \lambda_{J}$ and the integral term in Eq. (19) is smaller than $\varphi_{\omega}(0)$ by the parameter $\lambda / \lambda_{J}$. Therefore, we obtain

$$
E_{y}\left(0, k_{y}\right) \approx-\frac{\Phi_{0}}{2 \pi} i k_{\omega} \varphi_{\omega}(0), B_{z}\left(0, k_{y}\right) \approx-\frac{\Phi_{0}}{2 \pi} i \zeta k_{\omega} \varphi_{\omega}(0) .
$$

Within this approximation, the magnetic field at the junction edge which determines the boundary condition (12) is

$$
\begin{aligned}
& B_{z}(\mathbf{r}=0) \approx-\frac{\Phi_{0}}{2 \pi} i k_{\omega} \varphi_{\omega}(0) Z(\omega), \quad Z(\omega) \approx \\
& \int_{-\pi / d}^{\pi / d} \frac{d k_{y}}{2 \pi} \zeta\left(\omega, k_{y}\right) \approx \frac{\varepsilon_{d}\left|k_{\omega}\right|}{2}-i \frac{\varepsilon_{d} k_{\omega}}{\pi} \ln \frac{\pi / d}{\sqrt{\varepsilon_{d}}\left|k_{\omega}\right|} .
\end{aligned}
$$

Finally, we obtain the boundary conditions for smooth oscillating phase at both edges, $x=0,-l$, in a finite-length $\mathrm{JJ}$ :

$$
\nabla_{x} \varphi_{\omega}(x) \approx \pm 2 i \lambda k_{\omega} Z(\omega) \varphi_{\omega}(x), \text { for } x=0,-l
$$

Radiation outside $\mathrm{JJ}$ to the right is given by the Poynting vector, $\mathcal{P}_{\text {rad }}=$ $(c / 4 \pi) \int d y\left\langle E_{y}(0, y, t) B_{z}(0, y, t)\right\rangle_{t}$,

$$
\mathcal{P}_{\text {rad }} \approx \frac{\varepsilon_{d} w \omega^{3} \Phi_{0}^{2}}{64 \pi^{3} c^{2}}\left|\varphi_{\omega}(0)\right|^{2}
$$

We also present the phase equation and boundary conditions in the time representation. Introducing the dimensionless variables $\tau=\omega_{p} t$ and $u=x / \lambda_{J}$ and using Eq. (11), we write the equation for the total smooth phase $\varphi(\tau, u)$, which includes both oscillating and non-oscillating components

$$
\left[\frac{\partial^{2}}{\partial \tau^{2}}-\nabla_{u}^{2}+\left(\alpha_{t}-\alpha_{q} \nabla_{u}^{2}\right) \frac{\partial}{\partial \tau}\right] \varphi+\sin \varphi=0 .
$$

Using Eqs. (12), (21), and (22) and adding the boundary condition for the static phase, we obtain the dynamic boundary condition:

$$
\begin{aligned}
& \nabla_{u} \varphi(\tau, 0)=-b-\int_{-\infty}^{\tau} d \tau^{\prime} \mathcal{K}\left(\tau-\tau^{\prime}\right) \frac{\partial \varphi\left(\tau^{\prime}, 0\right)}{\partial \tau^{\prime}} \\
& \mathcal{K}(\tau)=\frac{d \varepsilon_{d}}{2 \pi \varepsilon_{i} \lambda_{J}} \frac{\partial}{\partial \tau}\left[\frac{\mathcal{F}(\xi \tau)}{\tau}\right], \mathcal{F}(v)=\int_{0}^{v} d z J_{0}(z) .
\end{aligned}
$$




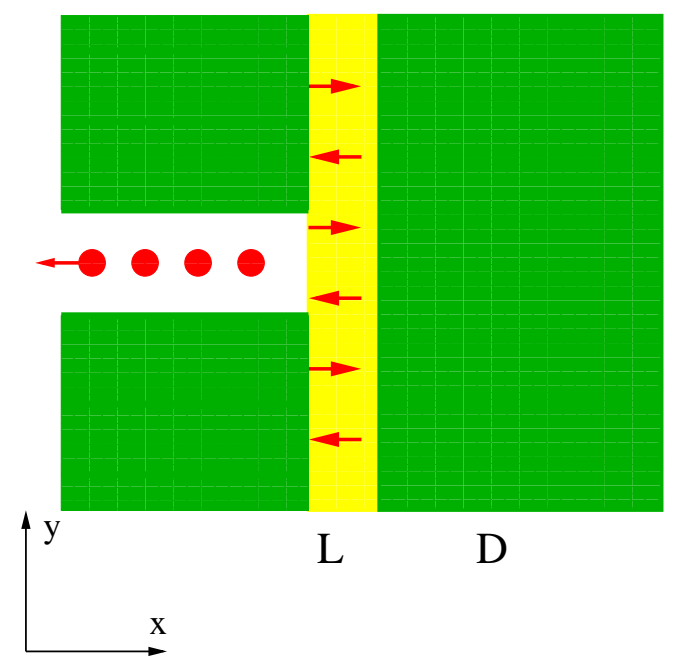

FIG. 2: The Josephson junctions at $x<0$, dielectric at $0<x<L \ll k_{\omega}^{-1}$ and superconducting screen with the London penetration length $\lambda_{s}$ and the thickness $D \gg \lambda_{s}$. Such a screen effectively reflects the Swihart wave back into the junction. It introduces additional interaction of vortices in different junctions in the case of multiple parallel junctions.

Here, $\xi=\pi c /\left(\omega_{p} d \sqrt{\varepsilon_{d}}\right), J_{0}(z)$ is the Bessel function, $b=4 \pi \lambda \lambda_{J} H_{0} / \Phi_{0}$ with $\mathbf{H}_{0} \| z$ being the applied dc magnetic field, $b \ll \lambda_{J} / \lambda$. For the edge $x=-l$ we need to change sign of $\mathcal{K}$. Due to the non-analytical $\omega$-dependence of $Z(\omega)$, the kernel $\mathcal{K}(\tau)$ is irregular at $d \rightarrow 0$ and then $\tau \rightarrow 0$. Singular frequency dependence of $Z(\omega)$ is due to retardation caused by electromagnetic wave propagation inside dielectric, Eq. (17). Thus boundary conditions also exhibit retardation effect.

\section{Outside superconducting screen}

Let us consider now a junction with superconducting screen at the right side separated from the layered crystal by the dielectric with the thickness $L$ small in comparison with the wave length of the electromagnetic wave in this dielectric, see Fig. 2. Such a screen prevents the radiation from the right side increasing it from other side. In the case of many parallel junctions the screen enhances their interaction as was suggested by Ivanchenko [11].

We consider the case $k_{\omega} L \ll 1$ and $L \ll \lambda_{s}$. Fields inside the superconducting screen obey 
Eq. (9). The solution is

$$
\begin{aligned}
& E_{y}(\omega, x, y)=\frac{1}{(2 \pi)^{2}} \int_{-\infty}^{+\infty} d k_{y} G\left(k_{y}\right) e^{-\kappa_{x} x+i k_{y} y}, \quad \kappa_{x}=\sqrt{\lambda_{s, \omega}^{-2}+k_{y}^{2}} \\
& E_{x}(\omega, x, y)=-\frac{1}{(2 \pi)^{2}} \int_{-\infty}^{+\infty} d k_{y} G\left(k_{y}\right) \frac{i k_{y}}{\kappa_{x}} e^{-\kappa_{x} x+i k_{y} y} \\
& B_{z}(\omega, x, y)=-\frac{1}{(2 \pi)^{2}} \int_{-\infty}^{+\infty} d k_{y} G\left(k_{y}\right) \frac{\lambda_{s, \omega}^{-2}}{i k_{\omega} \kappa_{x}} e^{-\kappa_{x} x+i k_{y} y}
\end{aligned}
$$

where $\lambda_{s, \omega}$, given by Eq. (4), characterizes the superconducting screen and $\lambda_{s}$ is the London penetration length of the screen. From this solution we obtain

$$
\begin{aligned}
& B_{z}(\omega, \mathbf{r}=0)=-\frac{\Phi_{0}}{2 \pi} i k_{\omega} \varphi_{\omega}(0) Z_{s}(\omega) \\
& Z_{s}(\omega)=-\frac{i}{\pi k_{\omega} \lambda_{s, \omega}^{2}} \ln \left(\frac{2 \lambda_{s}}{L}\right)
\end{aligned}
$$

This leads to the boundary condition

$$
\frac{\partial \varphi_{\omega}(0)}{\partial u}=\eta \varphi_{\omega}(0), \quad \eta \approx \frac{2 \lambda_{J} \lambda}{\pi \lambda_{s}^{2}} \ln \left(\frac{2 \lambda_{s}}{L}\right) .
$$

Note that $\eta \gg 1$. This results in the condition that $\varphi_{\omega}(0)$ is very close to zero for screened edge of the junction, while at the open edge the condition is that space derivative is close to zero. There is flow of energy from closed edge proportional to the small parameter $4 \pi \lambda_{s}^{2} \sigma_{q} / c$ due to quasiparticle dissipation inside the screen.

\section{Solution for the phase difference, $I-V$ characteristics, radiation and dissipation power}

Now we solve analytically Eq. (24) using the perturbation theory with respect to the Josephson current [32, 33] in the limit $|b-\tilde{\omega}| b \gg 1$. Taking solution as

$$
\varphi(\tau, u)=\tilde{\omega} \tau-b u+\theta(\tau, u), \quad \theta(\tau, u) \ll 1
$$

and expanding $\sin [\varphi(\tau, u)]$, we see that $\theta_{\omega}(u) \equiv \theta(\tilde{\omega}, u)$ obeys reduced versions of equation (11) with $s_{\omega}=-e^{i b u} / i$,

$$
\left[\nabla_{u}^{2}+\tilde{\omega}^{2}+i \tilde{\omega}\left(\alpha_{t}-\alpha_{s} \nabla_{u}^{2}\right)\right] \theta_{\omega}(u)=-e^{i b u} / i
$$

and boundary conditions (22)

$$
\frac{d \varphi}{d u}= \pm i \tilde{\omega} \beta \varphi, \text { for } u=0,-\tilde{l}
$$


with

$$
\beta=\beta_{0}\left(|\tilde{\omega}|-\frac{2 i \tilde{\omega}}{\pi} \ln \frac{\pi c / d}{\sqrt{\varepsilon_{d}} \omega_{p}|\tilde{\omega}|}\right), \beta_{0}=\frac{\varepsilon_{d} d}{2 \varepsilon_{i} \lambda_{J}}
$$

Solution for $\theta(\tilde{\omega}, u)$ given by,

$$
\theta_{\omega}(u) \approx-\frac{e^{i b u} / i}{\tilde{\omega}^{2}-b^{2}+i \alpha_{b} \tilde{\omega}}+a_{1} e^{i p_{\omega} u}+a_{2} e^{-i p_{\omega} u}
$$

describes the moving vortex lattice (the first term) and reflected Swihart waves propagating to the right and left. Here $\alpha_{b}=\alpha_{t}+\alpha_{q} b^{2}$ and $p_{\omega}=\sqrt{\left(\tilde{\omega}^{2}+i \alpha_{t} \tilde{\omega}\right) /\left(1-i \alpha_{q} \tilde{\omega}\right)} \approx \tilde{\omega}+i \alpha / 2$ with $\alpha=\alpha_{t}+\alpha_{q} \tilde{\omega}^{2}$. For a JJ with both open edges finding $a_{1}$ and $a_{2}$ from the boundary conditions (31) we obtain the phase distribution

$$
\begin{aligned}
& \theta_{\omega}(u)=-\frac{\exp (i b u) / i}{\tilde{\omega}^{2}-b^{2}+i \alpha_{b} \tilde{\omega}}- \\
& \frac{(b-\beta \tilde{\omega})\left[\cos \left[p_{\omega}(\tilde{l}+u)\right]-i \tilde{\beta} \sin \left[p_{\omega}(\tilde{l}+u)\right]\right]-(b+\beta \tilde{\omega}) \exp (-i b \tilde{l})\left[\cos \left(p_{\omega} u\right)+i \tilde{\beta} \sin \left(p_{\omega} u\right)\right]}{\left(\tilde{\omega}^{2}-b^{2}+i \alpha_{b} \tilde{\omega}\right) p_{\omega}\left[\left(1+\tilde{\beta}^{2}\right) \sin \left(p_{\omega} \tilde{l}\right)+2 i \tilde{\beta} \cos \left(p_{\omega} \tilde{l}\right)\right]}
\end{aligned}
$$

with $\tilde{\beta} \equiv \beta \tilde{\omega} / p_{\omega}$ and $\tilde{l}=l / \lambda_{J}$. Alternatively, the phase was obtained by expansion with respect to eigenmodes [32, 33]. In particular, the boundary values which determine outside irradiation are given by

$$
\begin{aligned}
\theta_{\omega}(0) & =\frac{i}{\tilde{\omega}^{2}-b^{2}+i \alpha_{b} \tilde{\omega}}\left[1-\frac{(b-\beta \tilde{\omega})\left[\cos \left(p_{\omega} \tilde{l}\right)-i \tilde{\beta} \sin \left(p_{\omega} \tilde{l}\right)\right]-(b+\beta \tilde{\omega}) \exp (-i b \tilde{l})}{i p_{\omega}\left[\left(1+\tilde{\beta}^{2}\right) \sin \left(p_{\omega} \tilde{l}\right)+2 i \tilde{\beta} \cos \left(p_{\omega} \tilde{l}\right)\right]}\right] \\
\theta_{\omega}(-\tilde{l}) & =\frac{i \exp (-i b \tilde{l})}{\tilde{\omega}^{2}-b^{2}+i \alpha_{b} \tilde{\omega}}\left[1-\frac{(b-\beta \tilde{\omega}) \exp (i b \tilde{l})-(b+\tilde{\beta} \tilde{\omega})\left[\cos \left(p_{\omega} \tilde{l}\right)-i \beta \sin \left(p_{\omega} \tilde{l}\right)\right]}{i p_{\omega}\left[\left(1+\tilde{\beta}^{2}\right) \sin \left(p_{\omega} \tilde{l}\right)+2 i \tilde{\beta} \cos \left(p_{\omega} \tilde{l}\right)\right]}\right]
\end{aligned}
$$

These cumbersome formulas can be significantly simplified in the regime $b \gg \tilde{\omega}$, weak dissipation $\alpha_{t}, \alpha_{q} \ll 1$, and large impedance mismatch, $|\beta| \ll 1$. In this case, keeping only Fiske resonance terms, we obtain

$$
\begin{aligned}
& \theta_{\omega}(0) \approx\left[\cos \left(p_{\omega} \tilde{l}\right)-\exp (-i b \tilde{l})\right] /(\tilde{\omega} b D), \\
& \theta_{\omega}(-l) \approx\left[1-\exp (-i b \tilde{l}) \cos \left(p_{\omega} \tilde{l}\right)\right] /(\tilde{\omega} b D), \\
& D \approx \sin (\tilde{\omega} \tilde{l})+i(2 \beta+\alpha \tilde{l} / 2) \cos (\tilde{\omega} \tilde{l})
\end{aligned}
$$

In these approximate results we also neglected the term $\operatorname{Im}[\beta(\omega)]$, which only slightly shifts resonance positions. Radiation to the right and left, $\mathcal{P}_{\text {rad }}^{r, l}(\tilde{\omega}, b)$, is determined by the values $\left|\theta_{\omega}(0)\right|^{2}$ 
and $\left|\theta_{\omega}(-l)\right|^{2}$. At low dissipation, $\alpha \tilde{l} \ll 1$, we derive

$$
\begin{aligned}
& \mathcal{P}_{\text {rad }}^{r, l} \approx \frac{\Phi_{0}^{2} \omega \omega_{p}^{2} w}{64 \pi^{3} c^{2} b^{2}} \frac{1-2 \cos (b \tilde{l}) \cos (\tilde{\omega} \tilde{l})+\cos ^{2}(\tilde{\omega} \tilde{l}) \pm \rho}{|D|^{2}}, \\
& \rho=2(\alpha \tilde{l}+2 \beta)[1-\cos (b \tilde{l}) \cos (\omega \tilde{l})] / b .
\end{aligned}
$$

The radiation reaches maxima for almost standing Swihart waves at frequencies $\tilde{\omega}=\tilde{\omega}_{n}=\pi n / \tilde{l}$ with $n=1,2, \ldots$ when $\cos (b \tilde{l}) \cos \left(\tilde{\omega}_{n} \tilde{l}\right) \neq 1$. The resonance width is determined by both, the dissipation, $\alpha \tilde{l}$, and by the radiation, $\beta$. The perturbation theory is valid in resonance for $\left|\theta_{\omega}(0)\right| \sim$ $(b \alpha \tilde{l} \tilde{\omega})^{-1}<1$ and the radiation power in this linear regime is quite small, $\mathcal{P}_{\mathrm{rad}} / w \lesssim 10^{-6}$ and $\lesssim 1 \mu \mathrm{W} / \mathrm{cm}$ at $\nu=10 \mathrm{GHz}$ and $1 \mathrm{THz}$, respectively. The asymmetry of radiation described by $\rho$ is small.

Next we derive the dc current at voltage $V=\Phi_{0} \omega /(2 \pi c)$ and estimate $Q$. The current $I$ via $\mathrm{JJ}$ is given by the tunneling quasiparticle contribution, $I_{t}=\sigma_{t} V l w / d$, and the Josephson current contribution, $I_{s}$,

$$
\begin{aligned}
I_{s}(\omega) & \approx J_{c} l w i_{J} \\
i_{J} & =\tilde{l}^{-1} \int_{-\tilde{l}}^{0} d u\langle\cos (\tilde{\omega} \tau-b u) \theta(\tau, u)\rangle_{\tau} .
\end{aligned}
$$

The exact result for the reduced Josephson current, $i_{J}$, is given by

$$
\begin{aligned}
i_{J} & =\frac{\alpha_{b} \tilde{\omega} / 2}{\left(\tilde{\omega}^{2}-b^{2}\right)^{2}+\alpha_{b}^{2} \tilde{\omega}^{2}} \\
& +\operatorname{Im}\left[\frac{\left(b^{2}+\tilde{\beta}^{2} p_{\omega}^{2}\right)\left[\cos (b \tilde{l})-\cos \left(p_{\omega} \tilde{l}\right)\right]+i \tilde{\beta}\left[\left(b^{2}+p_{\omega}^{2}\right) \sin \left(p_{\omega} \tilde{l}\right)-2 p_{\omega} b \sin (b \tilde{l})\right]}{\tilde{l} p_{\omega}\left(p_{\omega}^{2}-b^{2}\right)\left(\tilde{\omega}^{2}-b^{2}+i \alpha_{b} \tilde{\omega}\right)\left[\left(1+\tilde{\beta}^{2}\right) \sin \left(p_{\omega} \tilde{l}\right)+2 i \tilde{\beta} \cos \left(p_{\omega} \tilde{l}\right)\right]}\right]
\end{aligned}
$$

In the case of weak dissipation the total Josephson current can be split into the dissipation and radiation parts, $I_{s}=I_{s}$, dis $+I_{s}$, rad. The radiation part plays the same role as dissipation because in both cases energy is transfered from the moving vortex lattice to other degrees of freedom (to photons in the case of radiation). In the lowest order in $\lambda / \lambda_{J} \ll 1$ at the resonance frequencies we get

$$
I_{s, \text { dis }} \approx \frac{\alpha \varepsilon_{i} \omega_{p} l}{2 \varepsilon_{d} \omega d} I_{s, \operatorname{rad}} \approx \frac{\Phi_{0} c w \alpha \tilde{l} \sin ^{2}(b \tilde{l} / 2)}{32 \pi^{2} \lambda \lambda_{J} b^{2} \tilde{\omega}|D|^{2}} .
$$

Losses due to radiation are equivalent to those caused by a resistor with $R w=2 \pi /\left(\varepsilon_{d} \omega\right)$ attached parallel to $\mathrm{JJ}\left(R w \approx 90 \mathrm{ohm} \cdot \mathrm{cm}\right.$ for $\varepsilon_{d}=1$ and $\left.\nu=10 \mathrm{GHz}\right)$. The power fed into $\mathrm{JJ}$ is $\mathcal{P}=I V$. Part of it, $\left(I_{t}+I_{s}\right.$, dis $) V$, is dissipated inside $\mathrm{JJ}$, while another part, $I_{s, \text { rad }} V$, is radiated. Neglecting 
non-resonant part, $I_{t}$, we obtain for the radiated fraction, $Q \equiv \mathcal{P}_{\text {rad }} / \mathcal{P}$,

$$
Q=\operatorname{Re}[\beta] \frac{\tilde{\omega}}{2 \tilde{l}} \frac{\left|\theta_{\omega}(0)\right|^{2}+\left|\theta_{\omega}(-\tilde{l})\right|^{2}}{\alpha_{t} \tilde{\omega}+i_{J}}
$$

In the regime of weak dissipation and near resonances we approximately obtain

$$
Q \approx \frac{r}{1+r}, \quad r=\frac{I_{s, \mathrm{rad}}}{I_{s, \mathrm{dis}}}=\frac{2 \varepsilon_{d} d \omega}{\varepsilon_{i} \omega_{p} \alpha l} .
$$

To clarify the physical meaning of the parameter $r$, we can represent it as $r=Q_{Z}\left(w \gg k_{\omega}^{-1}\right) \mathcal{N}$ where

$$
Q_{Z}\left(w \gg k_{\omega}^{-1}\right)=\frac{2 \varepsilon_{d} d \omega}{\varepsilon_{i} \lambda_{J} \omega_{p}} \ll 1
$$

is the transmission coefficient of the electromagnetic wave at the junction edge (impedance mismatch) into free space in the limit $w k_{\omega} \gg 1$ and $\mathcal{N}=1 / \alpha \tilde{l}$ is the number of reflections before the Swihart wave decays inside JJ. At $w k_{\omega} \sim 1$ and our result is larger than that given by Eq. (1) by the factor $\mathcal{N}$. As dissipation inside JJ decreases $\left(\sigma_{t}\right.$ and $\sigma_{q}$ drop), $\mathcal{N}$ increases. As one can see from Eq. (40), the relation between the dissipative and radiative dampings is mainly determined by competition between the two small parameters, $\alpha$ and $d / l$. In the linear regime, $b \alpha \tilde{l} \tilde{\omega}>1$, we get $\mathcal{N}<b \tilde{\omega}$. Due to limitation $b<\lambda_{J} / \lambda$ we obtain $Q \lesssim(d / \lambda) \tilde{\omega}^{2} \sim 10^{-2}\left(\omega / \omega_{p}\right)^{2}$.

Figure 3 shows three-dimensional plots illustrating dependencies of the total reduced current density $J / J_{0}=\alpha_{t} \tilde{\omega}+i_{J}$ and the radiated fraction $Q$ on the Josephson frequency $\omega$ (voltage) and field $b$. For illustration purposes we used toy parameters, $\tilde{l}=4, \alpha_{t}=0.01, \alpha_{q}=0$, and $\beta_{0}=0.001$. Figure 4 shows voltage dependencies of $J$ and $Q$ for several fields. The current shows well-known sharp peaks at the Fiske-resonance frequencies, and peak amplitudes oscillate with the magnetic field [32, 33, 34]. The conversion coefficient $Q$ shows smooth oscillating behavior reaching maxima given by estimate (40) at the resonances. We also observe another nontrivial feature, $Q$ sharply drops at the position corresponding to the Eck resonance conditions, $\tilde{\omega}=b$, even though no feature is seen in $I-V$ dependencies for this frequency. For comparison we also plotted in Fig. 5 the same dependencies for the case of higher dissipation, $\alpha_{t}=0.1$. This case is quantitatively described by the linear approximation and allows us to trace switching between different Fiske peaks with increasing current.

For a JJ with closed and open edges we obtain

$$
\begin{aligned}
& \theta_{\omega}(0) \approx-\sin \left(p_{\omega} \tilde{l}\right) /(\tilde{\omega} b D), \\
& D \approx \cos (\tilde{\omega} \tilde{l})+(i / 2)(\beta+\alpha \tilde{l}) \sin (\tilde{\omega} \tilde{l}),
\end{aligned}
$$




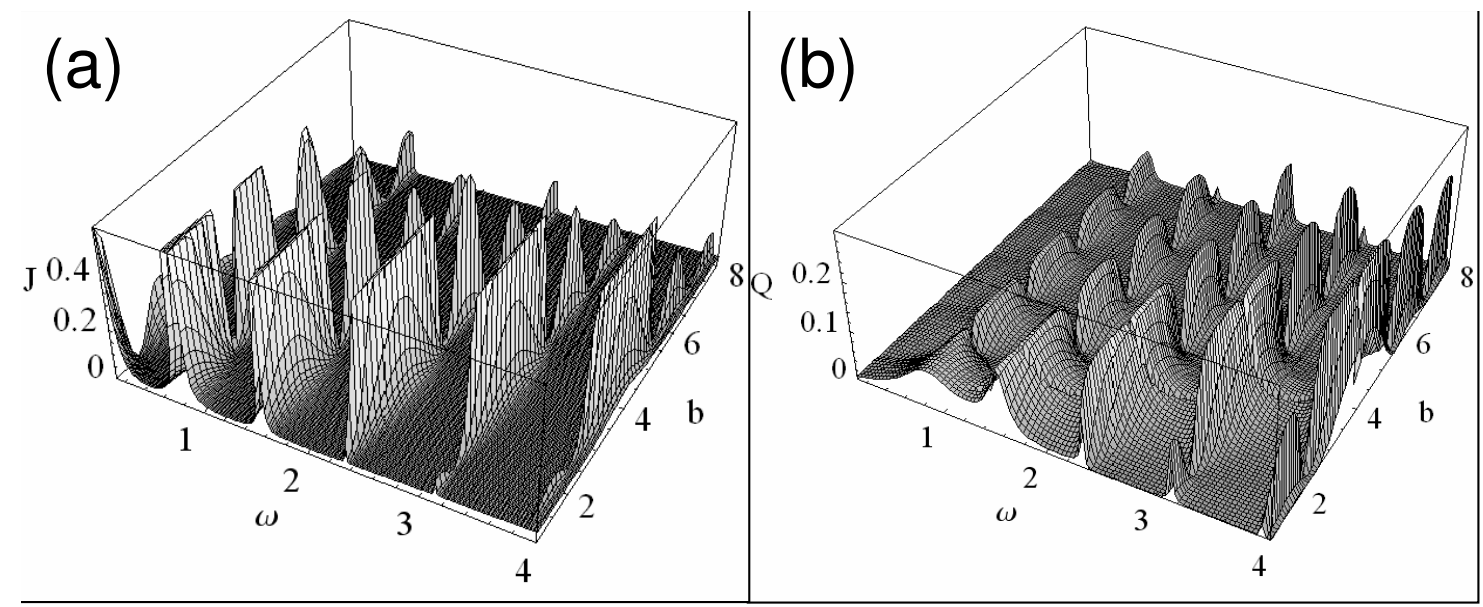

FIG. 3: Three-dimensional plots of the current density (left plot) and the radiated fraction (right plot) for $\tilde{l}=4, \alpha_{t}=0.01, \alpha_{q}=0$, and $\beta_{0}=0.001$ obtained within linear approximation.
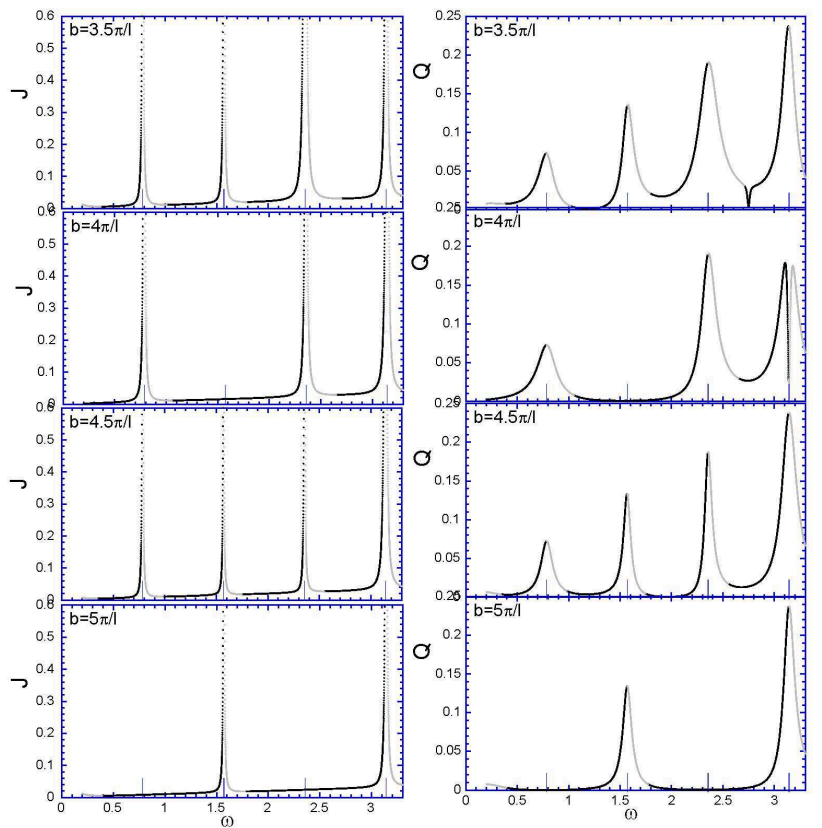

FIG. 4: The voltage dependencies of $J$ and $Q$ at several fields for the same parameters as in the previous plot. The current shows sharp increases near the marked Fiske-resonance frequencies. The linear approximation does not describe narrow regions near the resonances. $Q$ reaches maxima given by Eq. 40] at the resonances. In the plots for $b=3.5 \pi / l \approx 2.75$ and $4 \pi / l \approx 3.53$ one can see sharp drops of $Q$ at the frequencies, corresponding to the Eck resonance condition $\tilde{\omega}=b$. 


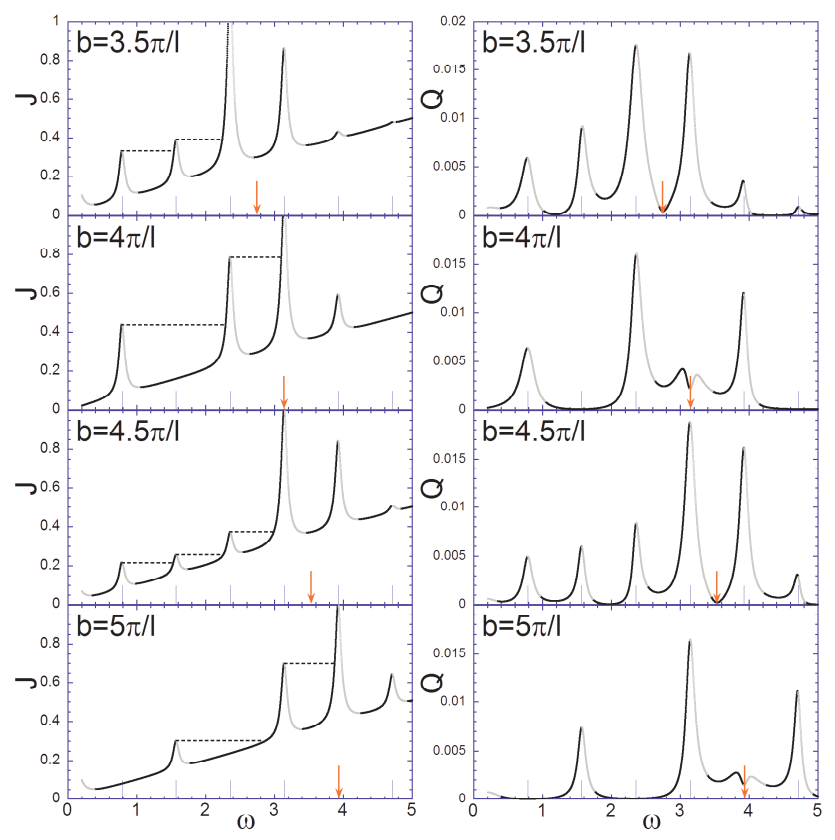

FIG. 5: The voltage dependencies of current $J$ and radiated fraction, $Q$, at several fields for the higher dissipation $\alpha_{t}=0.1$ (other parameters are the same as in the previous plots). Both $J$ and $Q$ show maxima near the marked resonance frequencies. Arrow marks frequency corresponding to the Eck resonance condition $\omega=b$. With current sweep the system will switch between different peaks, as it is illustrated by the dashed lines.

The Poynting vector of radiation from open edge at $x=0$ is given by the expression

$$
\mathcal{P}_{\text {rad }} \approx \frac{\Phi_{0}^{2} \omega \omega_{p}^{2} w}{64 \pi^{3} c^{2} b^{2}} \frac{\sin ^{2}(\tilde{\omega} \tilde{l})}{|D|^{2}},
$$

The $I-V$ characteristics defined by Eq. (35) consists of maxima at the frequencies $\omega_{n}$. The part of it, where $d I / d V<0$, is unstable in the current-biased regime. Experimentally only voltages corresponding to the resonance frequencies $\omega_{n}$ were observed [2, 3]. Numerical calculations [6, 7] show that such a behavior occurs in the nonlinear regime, i.e. at very low dissipation.

\section{Conclusions for single-junction case}

We have shown that in the case of weakly dissipative junctions $Q$ may become of order unity in the linear regime. Nevertheless, the radiation power per unit width, $\mathcal{P}_{\text {rad }} / w$, given by Eq. (23), is always small in this regime because the condition $\left|\theta_{\omega}\right| \lesssim 1$ also restricts the power fed into JJ. The open question is whether it is possible to get $\left|\theta_{\omega}\right| \gg 1$ and larger $\mathcal{P}_{\text {rad }}$ in strongly nonlinear regime 
when dissipation is very low and many Swihart modes are involved. The $I-V$ characteristics in this limit have the form of sharp steps (FIske steps) according to experimental data [2, 3] and numerical calculations [6, 7]. However, the amplitude $\varphi_{\omega}(0)$, which determines the radiation power, in highly nonlinear regime was not calculated yet.

In conclusion, accounting for the radiation into the dielectric outside media, we derived the dynamic boundary conditions for $\mathbf{J J}$ with the width $w$ much larger than the wave length of the electromagnetic wave radiated into the free space. The method of derivation may be extended to the case $w k_{\omega} \ll 1$, and following qualitative conclusions are valid for this case as well. We have shown that in the linear regime of Josephson oscillations the radiated fraction $Q$ of the power fed into the junction is determined by the number of multiple reflections (i.e. by dissipation rate inside $\mathbf{J J}$ ) and by the transmission coefficient, $Q_{Z}$, from $\mathbf{J J}$ into free space. Even if $Q$ reaches unity, radiation power per unit width of JJ remains small in the linear regime. To probe radiation power from JJ in the nonlinear regime, numerical study based on Eqs. (24) and (25) is needed. We think that measurements of radiation in the best junctions available now, like those studied in Ref. 35, at low temperatures and at intermediate magnetic fields may show higher radiation than that observed previously.

\section{RADIATION IN LAYERED SUPERCONDUCTORS}

For layered superconductors we need to formulate equations for the phase differences $\varphi_{n}(t, x)$ inside each intrinsic junction $n$ between layers $n$ and $n+1$ as well as the boundary conditions for these variables. Here coordinates of layers are $y=(n+1 / 2) s$ and layers are parallel to the plane $(x, z)$. In general $\varphi_{n}$ depend also on the coordinate $z$, the direction of the applied magnetic field $H_{0}$, but we will neglect this dependence (straight-vortex approach) assuming that width of the crystal along the $z$-axis is much larger than the radiation wavelength, $k_{\omega} w \gg 1$. The derivation of equations for $\varphi_{n}(t, x)[37,38,39]$ is similar to that for a single JJ. Using the Maxwell equations, the expression for the intralayer supercurrents via the phase of the superconducting order parameter inside layers and the Josephson relation for interlayer current one need to exclude all variables describing intralayer currents and electromagnetic fields induced by these currents by expressing them via $\varphi_{n}$. Formulation of the boundary conditions is also similar to a singlejunction case and is based on Eq. (16). However, solution of the equations for phase differences is now much more complicated because the vortex structure is two-dimensional (along $x$ and $y$ axis) 
and may vary depending on the parameters such as the applied dc magnetic field $H_{0}$, the length $l$ along the $x$-axis and also on the transport current (velocity of moving lattice). To have significant radiation power motion of vortex lattice in different intrinsic junction should be synchronized, as in multiple artificial JJ. For that interaction of intrinsic junctions should be strong enough and it should favor in-phase vortices in all junctions, i.e rectangular vortex lattice. First, we present the equations and the boundary conditions for $\varphi_{n}(t, x)$ and then discuss solutions for these equations and corresponding radiation power in linear regime of Josephson oscillations when perturbation theory may be used to solve equations for the phase differences. We will focus on crystals with large number of layers on the scale of the London penetration length $\lambda_{a b}$ for intralayer currents. In this case super-radiation from many layers becomes possible when there are many junctions on the scale of radiation wavelength and when vortices in many junctions are synchronized. We will show that in this case significant part of the energy fed into the crystal is converted into the radiation.

\section{Equations for the phase differences}

The crystal length $l$ is along the $x$-axis, while the transport current is perpendicular to the layers. Thus, the Josephson vortex lattice moves along the $x$ axis, see Fig. 6

As we assumed that there is no dependence of the phase difference, current density $\mathbf{j}$, and the electromagnetic fields on $z$ coordinate, the components $j_{z}, E_{z}, B_{x}$, and $B_{y}$ vanish. The phase difference $\varphi_{n}(t, x)$ between the layers $n$ and $n+1$ is defined as

$$
\varphi_{n}(t, x)=\Phi_{n}-\Phi_{n+1}-\frac{2 \pi}{\Phi_{0}} \int_{n s}^{(n+1) s} d y A_{y}(x)
$$

where $\Phi_{n}$ is the phase of the superconducting order parameter inside the layer. Intrinsic junction $n$ is in the space $(n-1 / 2) s<y<(n+1 / 2) s$. If all $N$ intrinsic Josephson junctions are identical then the dynamics of the system can be described by reduced coupled equations for the phase differences, $\varphi_{n}(t, x)$, and reduced magnetic fields $h_{n}=B_{y}(y=n s) / B_{c}$ with $B_{c} \equiv \Phi_{0} /\left(2 \pi \lambda_{a b} \lambda_{c}\right)$ (see. e.g., Ref. [41])

$$
\begin{aligned}
\frac{\partial^{2} \varphi_{n}}{\partial \tau^{2}}+\nu_{c} \frac{\partial \varphi_{n}}{\partial \tau}+\sin \varphi_{n}-\frac{\partial h_{n}}{\partial u} & =0 \\
\left(\nabla_{n}^{2}-\ell^{-2}\left(1+\nu_{a b} \frac{\partial}{\partial \tau}\right)\right) h_{n}+\left(1+\nu_{a b} \frac{\partial}{\partial \tau}\right) \frac{\partial \varphi_{n}}{\partial u} & =0
\end{aligned}
$$




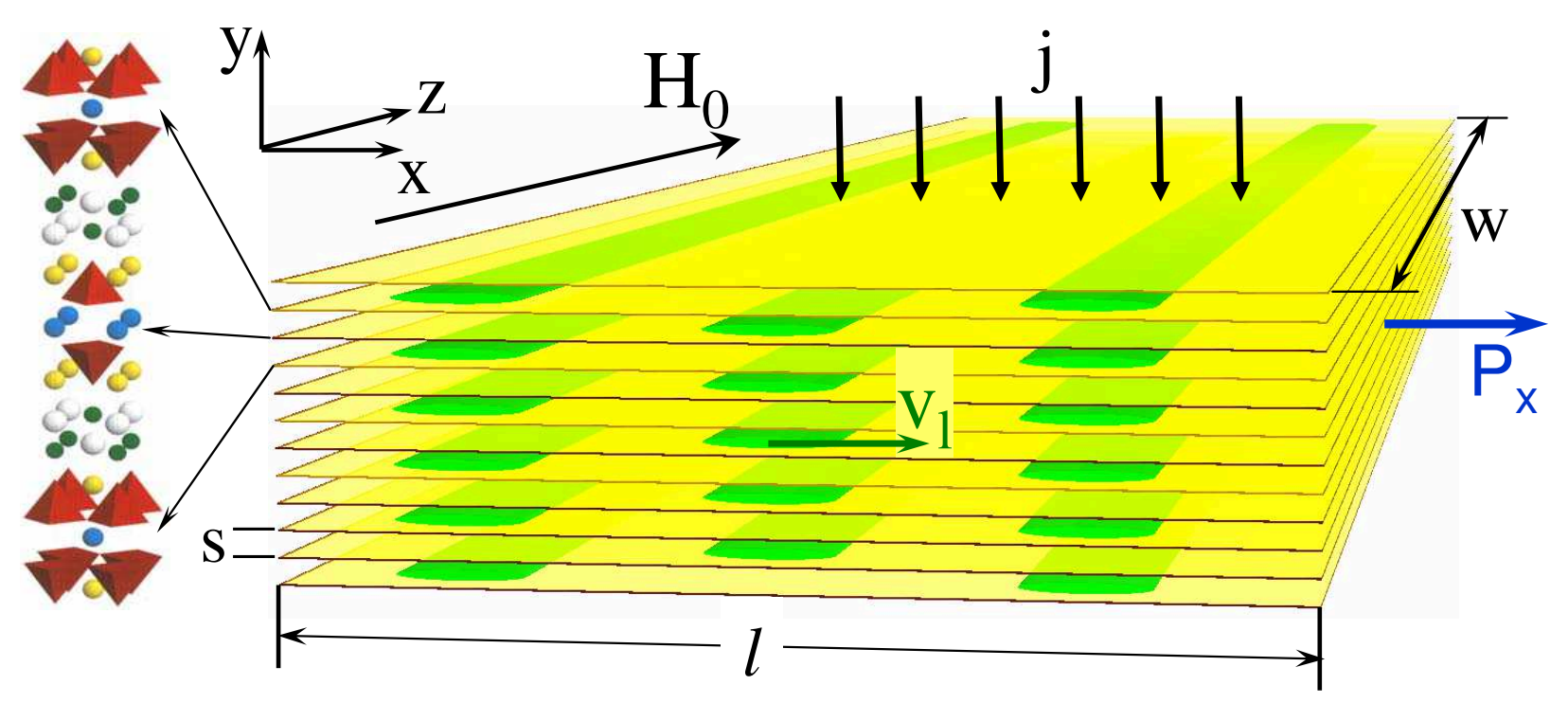

FIG. 6: Schematic picture of layered structure of superconductor and Josephson vortex lattice. The unit cell of $\mathrm{Bi}_{2} \mathrm{Sr}_{2} \mathrm{CaCu}_{2} \mathrm{O}_{8}$ compound and the relation of the crystal structure to the layered model are shown in the left. The directions of the applied dc magnetic field $H_{0}$, of the dc transport current $j$, and of the radiation Poynting vector $P_{x}$ are also shown. The Josephson vortices forming triangular lattice are shown schematically by elliptic cylinders and $v_{l}$ is the velocity of moving lattice.

Here we used reduced $x$ coordinate, $u=x / \lambda_{J}$ normalized to the Josephson length $\lambda_{J}=\gamma s$ and reduced time, $\tau=\omega_{p} t$, where $\omega_{p}=c /\left(\lambda_{c} \sqrt{\epsilon_{c}}\right)$ is the plasma frequency, $\epsilon_{c}$ is the $c$-axis highfrequency dielectric constant inside the superconductor, $\lambda_{a b}$ and $\lambda_{c}$ are the London penetration lengths, $\ell \equiv \lambda_{a b} / s, s$ is the interlayer distance, and $\nabla_{n}^{2}$ notates the discrete second derivative operator, $\nabla_{n}^{2} A_{n}=A_{n+1}+A_{n-1}-2 A_{n}$. The dissipation parameters, $\nu_{a b}=4 \pi \sigma_{a b} /\left(\gamma^{2} \epsilon_{c} \omega_{p}\right)$ and $\nu_{c}=4 \pi \sigma_{c} /\left(\epsilon_{c} \omega_{p}\right)$, are determined to the quasiparticle conductivities, $\sigma_{a b}$ and $\sigma_{c}$, along and perpendicular to the layers, respectively. The conductivity $\sigma_{a b}$ plays the same role as the conductivity $\sigma_{q}$ for a single JJ. Applying the operator $\nabla_{n}^{2}-\ell^{-2}\left(1+\nu_{a b} \frac{\partial}{\partial \tau}\right)$ to the first equation and excluding $h_{n}$, we can also derive equations containing only $\varphi_{n}(u, \tau)$

$$
\left[\nabla_{n}^{2}-\ell^{-2}\left(1+\nu_{a b} \frac{\partial}{\partial \tau}\right)\right]\left(\hat{T}_{c} \frac{\partial \varphi_{n}}{\partial \tau}+\sin \varphi_{n}\right)+\left(1+\nu_{a b} \frac{\partial}{\partial \tau}\right) \frac{\partial^{2} \varphi_{n}}{\partial u^{2}}=0,
$$

with $\hat{T}_{c} \equiv \partial / \partial \tau+\nu_{c}$. Eqs. (46) represent just charge conservation laws. The first term describes the change of electron charges inside the layers and Cooper-pair interlayer tunneling currents, while the second term describes superconducting intralayer currents. The terms with the coefficient $\nu_{a b}$ describe quasiparticle dissipative in-plane currents induced by moving Josephson vortices. The 
static interaction of junctions is described by the term $\nabla_{n}^{2} \sin \varphi_{n}$, while their dynamic interaction is described by the term $T_{c}(\partial / \partial \tau) \nabla_{n}^{2} \varphi_{n}$. Both are short-range (nearest-neighbor) weak interactions and they are not very effective in keeping long-range ordering of vortex lattice along the $y$-axis.

In the system of finite number of layers $N$ the dc current with the density $J$ is injected in layer 1 and extracted from layer $N$. Then equations for the first and the last junction are obtained from Eq. (46) by putting $\varphi_{0}=\varphi_{N+1}=0$ in linear terms and replacing $\sin \varphi_{0}=\sin \varphi_{N+1}=j$. In a finite-layer system the edge junctions differ strongly from other junctions because they have only one neighboring junction.

The electric field inside the superconductor between layers $n$ and $n+1$ in terms of the phase difference is given as

$$
E_{y ; n, n+1} \approx \frac{\Phi_{0}}{2 \pi s c} \frac{\partial \varphi_{n}}{\partial t} .
$$

The average (over time or space) electric field determines the Josephson frequency $\omega_{J}$. The average magnetic field we denote by $B$ and we introduce dimensionless average magnetic field $b=2 \pi s \lambda_{J} B / \Phi_{0}$.

The parameters of BSCCO at low temperatures are $\omega_{p} /(2 \pi)=0.15 \mathrm{THz}$, the Josephson critical current $J_{c}=1700 \mathrm{~A} / \mathrm{cm}^{2}, \gamma=500, \epsilon_{c}=12, s=15.6 \AA, \sigma_{c}(0)=2 \cdot 10^{-3}(\mathrm{ohm} \cdot \mathrm{cm})^{-1}$, $\sigma_{a b}(0)=4 \cdot 10^{4}(\mathrm{ohm} \cdot \mathrm{cm})^{-1}[45]$ and so $\ell \approx 100, \nu_{a b} \approx 0.2$ and $\nu_{c} \approx 5 \cdot 10^{-4}$. An important feature of the high-temperature superconductors is higher relative in-plane dissipation in comparison with $c$ axis dissipation, $\nu_{a b} \gg \nu_{c}$. Another layered high- $\mathrm{T}_{c}$ compound $\mathrm{Tl}_{2} \mathrm{Ba}_{2} \mathrm{CaCu}_{2} \mathrm{O}_{8}$ has lower anisotropy $\gamma \approx 150$ and as a consequence higher critical current $J_{c} \approx 3 \cdot 10^{4} \mathrm{~A} / \mathrm{cm}^{2}$ and higher plasma frequency, $\omega_{p} /(2 \pi) \approx 0.75 \mathrm{THz}[46]$.

\section{Boundary conditions and the Poynting vector of radiation}

We assume that there are only outcoming waves and use Eq. 16 for an open edge. In the junction $n$ we approximate $B_{z}(y) \approx B_{z}\left(y_{n}\right)$ and $E_{y}(y) \approx E_{y}\left(y_{n}\right)$, where $y_{n}=s n$. This gives the following boundary condition at the boundary $x=0$,

$$
\begin{aligned}
& B_{z}(\omega, n)=\sum_{m} U(\omega, n-m) E_{y}(\omega, m), \\
& U(\omega, n) \approx(1 / 2) s\left[\left|k_{\omega}\right| J_{0}\left(k_{\omega} s n\right)+i k_{\omega} N_{0}\left(k_{\omega} s n\right)\right], \quad n \neq 0,
\end{aligned}
$$




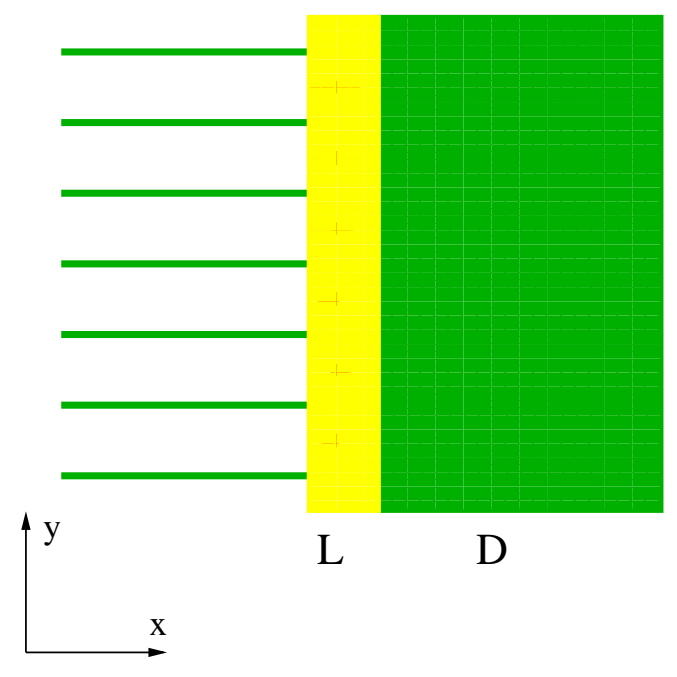

FIG. 7: The stack of intrinsic Josephson junctions at $x<0$, dielectric at $0<x<L \ll k_{\omega}^{-1}$ and a superconducting screen with the London penetration length $\lambda_{s}$ and the thickness $D \gg \lambda_{s}$. Such a screen introduces additional interaction of vortices in different intrinsic junctions.

while for $n=0$ we need to substitute $-(2 / \pi) \ln \left[1 /\left(\left|k_{\omega}\right| s\right)\right]$ for $N_{0}\left(k_{\omega} s n\right)$. Finally, we use the relations

$$
B_{z}(t, n)=B_{c} \ell^{2}\left[\frac{\partial \varphi_{n}}{\partial u}\right]_{x=0}, E_{y}(t, n)=\frac{B_{c} \ell}{\sqrt{\epsilon_{c}}}\left[\frac{\partial \varphi_{n}}{\partial \tau}\right]_{x=0}, B_{c}=\frac{\Phi_{0}}{2 \pi \lambda_{a b} \lambda_{c}}
$$

to formulate the boundary condition for oscillating part of $\varphi_{n}(x, t)$. This gives the following general boundary conditions for the phase differences

$$
\frac{\partial \varphi_{n}}{\partial u}= \pm \frac{i s \tilde{\omega}}{2 \ell \sqrt{\varepsilon_{c}}} \sum_{m}\left[\left|k_{\omega}\right| J_{0}\left(k_{\omega} s|n-m|\right)+i k_{\omega} N_{0}\left(k_{\omega} s|n-m|\right)\right] \varphi_{m}
$$

In the time representation we obtain again Eq. (17), as for a single junction.

For the Poynting vector we derive

$$
P_{x}(\omega)=\frac{\Phi_{0}^{2} \omega^{3}}{64 \pi^{3} c^{2} N s} \sum_{n, m} J_{0}\left(k_{\omega} s|n-m|\right) \varphi_{n}(\omega, 0) \varphi_{m}^{*}(\omega, 0) .
$$

Let us consider now the layered superconductor with a superconducting screen at the right side separated from the layered crystal by the dielectric with thickness $L$ small in comparison with the wavelength of the electromagnetic wave in this dielectric, see Fig. 7

Then, as in the case of a single JJ, we neglect effect of the dielectric on the boundary conditions. The solution inside the superconducting screen is given by Eq. (26). From this solution we obtain

$$
B_{z}(\omega, y)=\frac{i \lambda_{s, \omega}^{-2}}{k_{\omega}} \int d y^{\prime} K_{0}\left(\frac{y-y^{\prime}}{\lambda_{s, \omega}}\right) E_{y}\left(\omega, y^{\prime}\right) .
$$


This relation leads to the boundary condition for the oscillating part of the phase difference

$$
\frac{\partial \varphi_{n}(\omega, 0)}{\partial u}=\frac{\lambda_{J} s}{\lambda_{s, \omega}^{2}} \sum_{m} K_{0}\left[\frac{s(n-m)}{\lambda_{s, \omega}}\right] \varphi_{m}(\omega, 0) .
$$

Here space derivative of the phase differences and the phase differences are at the point $x \rightarrow-0$. The space derivative now is not small, the parameter $\left(s \lambda_{J} / \lambda_{s}^{2}\right)$ may be of order unity or larger. The term in the free energy, $\mathcal{F}_{b c}$, corresponding to the boundary condition Eq. (53), is

$$
\begin{aligned}
& \frac{\mathcal{F}_{b c}}{w}=\int d y \int d x \frac{B_{z}^{2}}{4 \pi}=\lambda_{s, \omega} s \sum_{n} \frac{B_{z}^{2}(s n)}{4 \pi}= \\
& =\frac{\Phi_{0}^{2} s}{16 \pi^{3} \lambda_{s, \omega}^{3}} \sum_{n, m} H\left[\frac{s(n-m)}{\lambda_{s, \omega}}\right] \varphi_{n}(\omega, 0) \varphi_{m}^{*}(\omega, 0), \quad H(a n)=\sum_{k} K_{0}(a k) K_{0}[a(k+n)] .
\end{aligned}
$$

This free energy can be compared with the bulk inductive interaction, corresponding to Eq. (46), see Ref. 40 ,

$$
\mathcal{F}_{\text {ind }}=\frac{\Phi_{0}^{2}}{32 \pi^{3} \lambda_{a b} \lambda_{c}} \int d u \nabla_{u} \varphi_{n}(u) \nabla_{u} \varphi_{m}(u) \exp \left[-\frac{|n-m| s}{\lambda_{a b}}\right] .
$$

Both interactions favor triangular lattice at least in the static case. The coefficient in Eq. (54) is much larger than that in the inductive free energy, i.e. superconducting screen enhances strongly the tendency to form triangular lattice along the $y$-axis for not very large junction length $l$.

\section{Solutions for the phase difference and the radiation power at high fields in large- $N$ case}

We consider here the simplest case of layered crystals with large number of layers $N \gtrsim \ell$, when we can neglect edge effects along the $y$-axis, i.e. the difference between the edge (the first and the last) and inner junctions. This corresponds to the thickness larger than $0.2 \mu \mathrm{m}$ with the total number of junctions $N>100$.

In the linear regime of Josephson oscillations the general solution for the phase difference has the form

$$
\varphi_{n}(\tau, u)=\tilde{\omega} \tau-b u+\kappa_{n}+\theta_{n}(\tau, u), \theta_{n}(\tau, u) \ll 1 .
$$

For $\kappa_{n}=0$ the lattice is rectangular, $\varphi_{n}(\tau, u)$ are $n$-independent. For $\kappa_{n}=\pi n$ the lattice is triangular, vortices in neighboring layers are in anti-phase positions. For a static lattice such a configuration minimizes the energy of the magnetic field inside the crystal with large $l, N$. However, at small $l$ boundary conditions are inconsistent with triangular lattice at some values of $b l$, and in this case rectangular lattice becomes more favorable [43]. For moving vortex lattice energy 
consideration does not work, and here the parameters $\kappa_{n}$ should be determined by the condition that total current $I$ via each junction is the same [41],

$$
J_{c} \lambda_{J} w \int_{-\tilde{l}}^{0} d u\left\langle\sin \varphi_{n}(\tau, u)\right\rangle_{\tau}=I, \quad .
$$

where $J_{c}=c \Phi_{0} /\left(8 \pi^{2} s \lambda_{c}^{2}\right)$ is the Josephson current density. Slowly moving lattice preserves its triangular structure. This was confirmed experimentally via observation of magnetic oscillations of the flux-flow resistivity with the period of one flux quantum per two junctions [44]. Theoretical analysis [41, 42] shows that the triangular lattice becomes unstable at the lattice velocity slightly smaller than the Swihart velocity $c_{S}=c s /\left(2 \lambda_{a b} \sqrt{\epsilon}\right)$. This instability corresponds to experimentally observed end point of the first flux-flow branch. It was also shown that interaction with top and bottom surfaces leads to significant lattice deformations [41]].

Situation at high velocity $v_{l} \gg c_{S}$ is less clear. Lattice structures and their stability in this regime in the case of large lateral size $l$ have been addressed in Ref. [41] and have been reconsidered in Ref. [42] with the conclusion that for parameters typical for BSCCO no stable regular lattice exists at high velocities. Structures and stability of steady states for small lateral sizes $l$ is an open issue.

In the following sections we will estimate the radiation power for rectangular and triangular lattice and for lattice with random values of $\kappa_{n}$. Those are most probable realizations of vortex configurations in the large $N$ limit. They give also an estimate for maximum radiation power which one can anticipate.

\section{Rectangular vortex lattice}

For rectangular lattice sketched at Fig. $8, \varphi_{n}=\tilde{\omega} \tau-b u+\theta(\tau, u)$, the equation for the oscillating part, $\theta(u)$, is given by

$$
\frac{\partial^{2} \theta}{\partial \tau^{2}}+\nu_{c} \frac{\partial \theta}{\partial \tau}-\ell^{2} \frac{\partial^{2} \theta}{\partial u^{2}} \approx-\sin (\tilde{\omega} \tau-b u) .
$$

For a junction opened at both edges the solution is similar to that of a single JJ, Eq. (32). However, there is important difference due to the presence of large coefficient $\ell^{2}$ in front of the second space derivative. This coefficient is due to uniformity of rectangular lattice and corresponding supercurrents along the $y$-axis, see Fig. 8. This uniformity leads to large energy of the magnetic field, i.e. inductive coupling, Eq. (55), resulting in small amplitude of phase variations. To find radiation power and $I-V$ characteristics we use results of the perturbation theory for a single JJ. To 


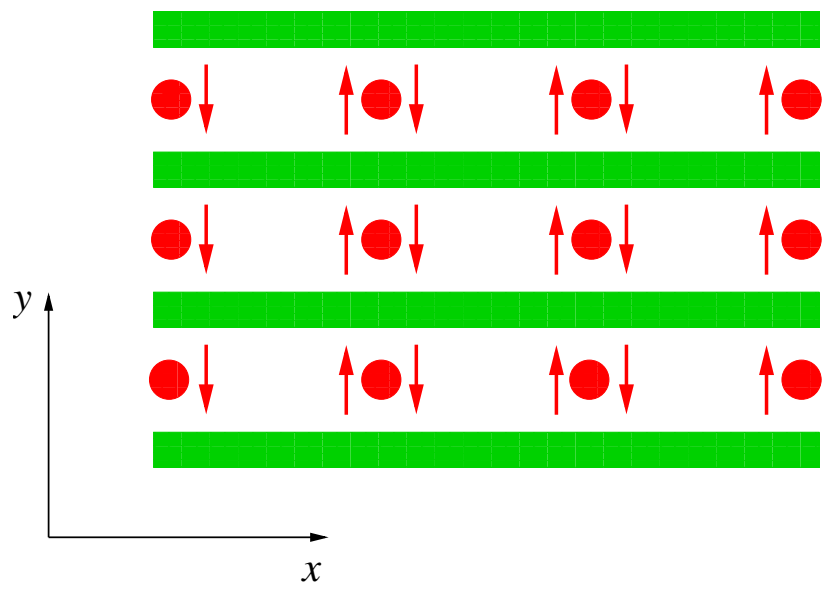

FIG. 8: Rectangular lattice of Josephson vortices in layered superconductors. The screening currents flow along the $y$ axis leading to the large energy of the magnetic field (inductive coupling). Correspondingly, the amplitude of phase modulation is weak, but vortices come to the junction edges coherently inducing super-radiation.

make Eq. (58) similar to that for a single JJ we introduce $\tilde{u}=u / \ell, \tilde{b}=b \ell$ and $\tilde{l}$ should be replaced by $\tilde{l} / \ell$. We limit ourself to the frequencies much smaller than the Eck resonance $\tilde{\omega} \ll b \ell$. In this case the solution has the form $\theta(\tau, \tilde{u})=\operatorname{Re}\left[\theta_{\omega} \exp (-i \omega \tau)\right]$ with

$$
\theta_{\omega}(u) \approx e^{i \tilde{b} \tilde{u}} /\left(i \tilde{b}^{2}\right)+a_{1} \exp \left(i p_{\omega} \tilde{u}\right)+a_{2} \exp \left(-i p_{\omega} \tilde{u}\right)
$$

The coefficients $a_{1}$ and $a_{2}$ should be found from the boundary conditions given by Eq. (50). For rectangular lattice at the edge $x=0$ we obtain

$$
\frac{\partial \theta_{\omega}(0)}{\partial \tilde{u}}=\frac{i \tilde{\omega}}{2 \sqrt{\epsilon_{c}}} s \sum_{n}\left[\left|k_{\omega}\right| J_{0}\left(k_{\omega} s n\right)+i k_{\omega} N_{0}\left(k_{\omega} s n\right)\right] \theta_{\omega}(0) .
$$

Large number of junctions $N>\left(k_{\omega} s\right)^{-1}$

We consider first the case of very large number of layers $N>\left(k_{\omega} s\right)^{-1}$. Then

$$
\frac{\partial \theta_{\omega}(0)}{\partial \tilde{u}} \approx \frac{i \tilde{\omega}}{\sqrt{\epsilon_{c}}} \theta_{\omega}(0)
$$

This corresponds to $\beta \approx 1 / \sqrt{\epsilon_{c}}$. Important point is that for such $\beta$ the perturbation theory is correct for $\tilde{\omega}|b \ell-\tilde{\omega}| \gg 1$, i.e. practically at all interesting fields and frequencies. We limit ourself to the frequencies smaller than the Eck resonance $\tilde{\omega} \ll b$. Thus the amplitude of Swihart 
waves is proportional to small factor $\ell^{-1}$ and oscillating part of the phase differences at $N s k_{\omega} \gtrsim 1$ is described by Eqs. (33) with the extra factor $\ell^{-1}$ for $\theta_{\omega}(0)$ and $\theta_{\omega}(-l)$. Further, we need to replace $\tilde{\omega} \tilde{l}$ by $\tilde{\omega} \tilde{l} / \ell \approx 0$, while in $D$ we need to put $\beta=1 / \sqrt{\epsilon_{c}}$ and $\alpha=\nu_{c}$. Assuming that the length of junctions is small enough, $\tilde{\omega} \tilde{l} \ll \ell$ and using results Eq. (33) we see that oscillatory dependence of the radiation power and of the dc current on $\tilde{\omega} \tilde{l}$ drops out and we obtain for the dc current at $\tilde{\omega} b \ell \gg 1$ and $\tilde{\omega} \tilde{l} \ll \ell$ the expression without resonances

$$
i=\frac{I}{J_{c} w l}=\nu_{c} \tilde{\omega}+\frac{\sqrt{\epsilon_{c}}|\sin (b \tilde{l} / 2)|}{b^{2} \ell \tilde{l}} \frac{1}{\tilde{\omega}} .
$$

For the radiation power of $N$ layers, using Eq. (51), we obtain

$$
\frac{\mathcal{P}_{\mathrm{rad}}}{w} \approx \frac{\Phi_{0}^{2} \omega_{p}^{2} \epsilon_{c}}{32 \pi^{3} c b^{2}} \frac{N}{s \ell^{2}} \sin ^{2}(b \tilde{l} / 2)
$$

Comparing this result with that for a single junction, Eq. 34, we see that the additional factor $N /\left(k_{\omega} s \ell^{2}\right)$ is present. In BSCCO and TBCCO crystals at the frequency $\nu=1 \mathrm{THz}$ the parameter $k_{\omega} s \ell^{2} \sim 1$. Thus the radiation power of $N$ layers for a moving rectangular lattice is $N /\left(k_{\omega} s\right)$ times larger than from a single intrinsic junction and additional large factor $1 /\left(k_{\omega} s\right)$ is due to super-radiation in the case $N k_{\omega} s \gtrsim 1$.

Let us discuss now the $I-V$ characteristics given by Eq. (62). At a given current $i>i_{\min }$ we have stable solution with positive slope, $d I / d V>0$, at $\tilde{\omega}>\tilde{\omega}_{\min }$. Here

$$
\tilde{\omega}_{\min }=\left(\frac{\sqrt{\epsilon_{c}}}{b^{2} \ell \tilde{l} \nu_{c}}\right)^{1 / 2}|\sin (b \tilde{l} / 2)|, \quad i_{\min }=2 \nu_{c} \omega_{\text {min }} .
$$

Hence, moving rectangular lattice cannot exist at currents $i<i_{\min }$ due to super-radiation as power fed into the crystal should support radiation as well as quasiparticle dissipation needed to ensure stability of this dynamic state. This condition is necessary but it may be not sufficient for the stability of moving rectangular lattice. The ratio of radiation power to that of dissipation one is $r=\left(\tilde{\omega}_{\min } / \tilde{\omega}\right)^{2}$. Thus maximum value of $Q$ is 0.5 at $i=i_{\text {min }}$, and $Q$ drops as current increases beyond $i_{\min }$.

For BSCCO crystals at $\tilde{l}=\pi$ and $b=1$ we estimate $\tilde{\omega}_{\min } \approx 4$, while $i_{\min } \approx 4 \times 10^{-3}$. At the frequency $\nu=1 \mathrm{THz}$ we obtain $\mathcal{P}_{\text {rad }} / w \sim N(\mu \mathrm{W} / \mathrm{cm})$ for $\mathrm{Tl}_{2} \mathrm{Ba}_{2} \mathrm{CaCu}_{2} \mathrm{O}_{8}$ crystals. Using the energy conservation law, $I V=\mathcal{P}_{\text {rad }}$, we see that to reach this power the total dc current $I$ via the junction should be about 0.004 of the critical current. The radiation power in BSCCO crystals at the same frequency is about 25 times weaker due to smaller Josephson plasma frequency. 
Moderate number of junction $\ell<N \ll\left(k_{\omega} s\right)^{-1}$

Consider now practically more interesting case of moderate number of layers, $\ell<N \ll$ $\left(k_{\omega} s\right)^{-1}$. In this case from Eq. 60 we obtain the following boundary condition

$$
\begin{aligned}
\frac{\partial \theta_{\omega}(0)}{\partial u} & =\frac{i \tilde{\omega} \beta_{N}}{\ell} \theta_{\omega}(0), \\
\beta_{N} & =\frac{s N}{2 \sqrt{\epsilon_{c}}}\left[\left|k_{\omega}\right|-i \frac{2 k_{\omega}}{\pi} \ln \frac{1}{\left|k_{\omega}\right| s N}\right] .
\end{aligned}
$$

This condition is very similar to the boundary condition (22) for a single junction.

Solving for $a_{1}$ and $a_{2}$, we obtain for the edge phase

$$
\theta_{\omega}(0) \approx \frac{\cos (\tilde{\omega} \tilde{l} / \ell)-\exp (-i b \tilde{l})}{\ell b \tilde{\omega}\left[\sin (\tilde{\omega} \tilde{l} / \ell)+i\left(2 \beta_{N}+\nu_{c} \tilde{l} / 2 \ell\right) \cos (\tilde{\omega} \tilde{l} / \ell)\right]}
$$

Due to $\beta_{N} \ll 1$ at small dissipation we obtain the resonant-type $I$ - $V$ characteristics with Fiske resonances at $\tilde{\omega} \tilde{l} / \ell=\pi n$, as in a single JJ. Moreover, for parameters of BSCCO the c-axis quasiparticle dissipation is negligible in comparison with the radiation damping. At resonances we derive

$$
i_{J}=\nu_{c} \tilde{\omega}+\frac{1-(-1)^{n} \cos (b \tilde{l})}{2 \tilde{l} \tilde{\omega} \ell b^{2} \operatorname{Re}\left[\beta_{N}\right]},
$$

The linear approximation brakes down at $2 \ell b \tilde{\omega} \operatorname{Re} \beta_{N} \sim 1$, i.e., it is valid for number of layers $N>\sqrt{\epsilon_{c}} /\left(b^{2} \tilde{\omega} \lambda_{a b}\left|k_{\omega}\right|\right)$. At the frequency $1 \mathrm{THz}$ linear approximation is valid if $N>100$. The total radiation power per unit width at the resonance frequency does not depend on number of layers and is given by

$$
\frac{\mathcal{P}_{\mathrm{rad}}}{w}=\frac{\epsilon_{c} \Phi_{0}^{2} \omega_{p}^{2}}{32 \pi^{3} \lambda_{a b}^{2} \omega b^{2}}\left[1-(-1)^{n} \cos (b \tilde{l})\right] .
$$

The independence of this result on $N$ appears as a result of cancellation of the $N^{2}$ factor in the total power of $N$ coherently radiating junctions and the factor $\beta_{N}^{-2} \propto N^{-2}$ which determines the resonance damping. Away from resonances and for resonances limited by quasiparticle damping $\mathcal{P}_{\text {rad }} \propto N^{2}$. For BSCCO crystals at $\omega / 2 \pi=1 \mathrm{THz}$, we obtain $\mathcal{P}_{\text {rad }} / w \approx 24 \mathrm{~mW} / \mathrm{cm}$, while for $\mathrm{Tl}_{2} \mathrm{Ba}_{2} \mathrm{CaCu}_{2} \mathrm{O}_{8}$ crystals we estimate $\mathcal{P}_{\text {rad }} / w \approx 0.5 \mathrm{~W} / \mathrm{cm}$. Now $\tilde{\omega}_{\text {min }}$ and $i_{\text {min }}$ become larger as $N$ drops:

$$
\tilde{\omega}_{\min }^{3 / 2}=\left(\frac{\sqrt{\epsilon_{c}}}{b^{2} \ell \tilde{l} \nu_{c}}\right)^{1 / 2} \frac{2|\sin (b \tilde{l} / 2)|}{\sqrt{\omega_{p} s N / c}}, \quad i_{\min }=\frac{3}{2} \nu_{c} \omega_{\min } .
$$

As $N$ increases beyond $N \sim \ell$ the total radiation power does not depend on $N$ in Fiske resonances and increases $\propto N^{2}$ outside resonances, while $\tilde{\omega}_{\text {min }}$ decrease as $N^{-1 / 3}$. This behavior holds until $N$ reaches $\left(k_{\omega} s\right)^{-1}$. After that $\mathcal{P}_{\text {rad }}$ increases linearly with $N$, while $\tilde{\omega}_{\text {min }}$ remains constant. 
It is interesting to compare our estimate with recent large-scale simulations of $\mathrm{THz}$ radiation out of BSCCO mesa by Tachiki et al. [27]. They solve the Maxwell equations coupled with the equations for the intralayer phases inside the crystal and the dielectric media. They found that close to one of the Fiske resonances lattice is mostly disordered but with pronounced rectangular correlations promoted by generated standing electromagnetic wave. They found quite powerful outside radiation in this state, with power density up to $P_{x}=3000 \mathrm{~W} / \mathrm{cm}^{2}$. Our estimate following from Eq. 69) for 100 junctions occurs to be only slightly smaller $\sim 1500 \mathrm{~W} / \mathrm{cm}^{2}$.

\section{Triangular lattice}

Triangular lattice, again in the limit $b \gg 1$, behaves quite differently in comparison with rectangular one. The solution has the form

$$
\varphi_{n}(\tau, u)=\tilde{\omega} \tau-b u+\pi n+(-1)^{n} \phi(\tau, u)+\theta(\tau, u) .
$$

Here $\phi$ describes the amplitude of the phase oscillations with Josephson frequency $\omega$, while $\theta$ describes oscillations with the frequency $2 \omega$ which are induced by moving vortex lattice due to nonlinearity of coupled sine-Gordon equations [46, see Ref. 26. According to theoretical estimates [41] when the lattice velocity approaches the Swihart velocity, the lattice may generate a very powerful electromagnetic wave inside superconductor, with power density up to $20 \mathrm{~W} / \mathrm{cm}^{2}$. Unfortunately, this main harmonic of electromagnetic wave at the frequency $\omega$ experiences full internal reflection at the boundary and does not radiate outside. The triangular lattice produce only weak outside radiation at frequency $2 \omega$, caused by the homogeneous in c direction component of the phase, $\theta(\tau, u)$. In the case of semiinfinite superconductor the radiation power at $2 \omega$ has been estimated in by Artemenko and Remizov [26]. Here we estimate this power for finite-size samples when pronounced Fiske resonances are present.

Putting the solution Eq. (71) into Eq. (46) we obtain coupled equations for $\phi(\tau, u)$ and $\theta(\tau, u)$ :

$$
\begin{aligned}
& \frac{\partial^{2} \phi}{\partial \tau^{2}}+\frac{\partial}{\partial \tau}\left(\nu_{c}-\frac{\nu_{a b}}{4} \frac{\partial^{2}}{\partial u^{2}}\right) \phi+\frac{1}{4} \frac{\partial^{2} \phi}{\partial u^{2}}=-\sin (\tilde{\omega} \tau-b u), \\
& \frac{\partial^{2} \theta}{\partial \tau^{2}}+\nu_{c} \frac{\partial \theta}{\partial \tau}-\ell^{2} \frac{\partial^{2} \theta}{\partial u^{2}}=-\phi \cos (\tilde{\omega} \tau-b u),
\end{aligned}
$$

From the second equation we see that $\theta$ is of order $\ell^{-1}$ and, as a consequence, terms linear in $\theta$ were omitted in the first equation in comparison with the term describing dissipation. 


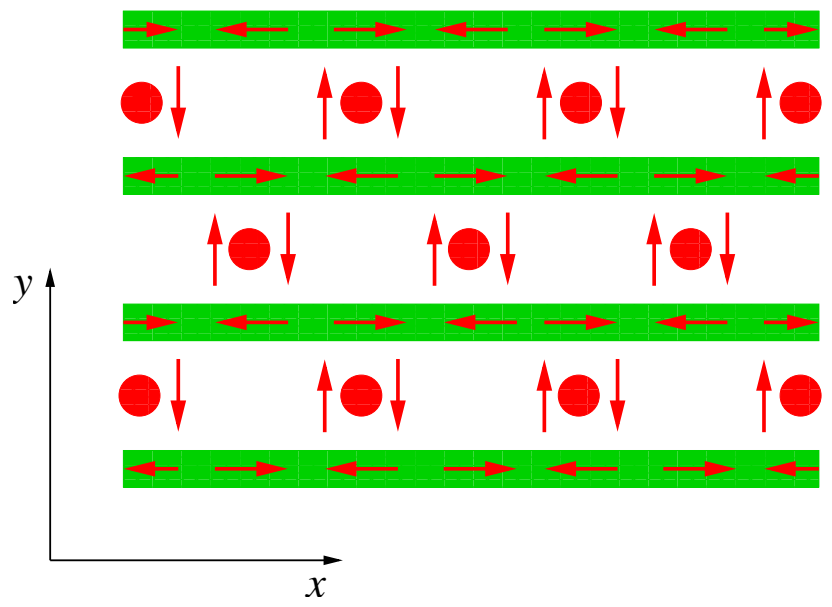

FIG. 9: Triangular lattice of Josephson vortices in layered superconductors. Screening currents compensate each other inside the lattice vortex cell $(2 s, 1 / b)$ leading to significant reduction of the magnetic energy and enhancement of the amplitude of the phase variations in comparison with rectangular lattice. The in-plane screening currents lead to intralayer quasiparticles contribution to the dissipation of the moving lattice. Due to nonlinearity there are field components at the frequency $2 \omega$ with small amplitude. They cause superradiation at the frequency $2 \omega$.

The boundary conditions for the alternating and homogeneous phase are given by

$$
\frac{\partial \phi(0)}{\partial u}=0, \quad \frac{\partial \theta(0)}{\partial u} \approx \pm i \beta \tilde{\omega} \theta(0)
$$

for $u=0, \tilde{l}$ with $\beta=1 / \sqrt{\epsilon_{c}}$. In the framework of the perturbation theory, at $\tilde{\omega} b \alpha_{\operatorname{tr}} \tilde{l} \gtrsim 1$, the amplitude $\phi$, in the lowest order in $1 / b$, is given by the expression

$$
\phi=\frac{4[\cos (q(u+l))-\exp (-i b l) \cos (q u)]}{b q \sin (q l)}, q=2 \tilde{\omega}+\alpha_{\mathrm{tr}}
$$

where $\alpha_{\mathrm{tr}}=\nu_{c}+\nu_{a b} \tilde{\omega}^{2} / 4$. Putting it into the equation for $\theta$ and accounting for boundary conditions we obtain

$$
\theta \approx \frac{i}{2 \tilde{\omega} \beta \ell b^{3}}[1-\exp (-2 i b \tilde{l})]-\frac{[\cos (2 \tilde{\omega} \tilde{l})-\exp (-i \tilde{b})][1-\exp (-i b \tilde{l})]}{\omega^{2} \beta \ell b^{2}\left[\sin (2 \tilde{\omega} l)+i \alpha_{\operatorname{tr}} \tilde{l} \cos (2 \tilde{\omega} \tilde{l})\right]} .
$$

Keeping only the resonance term, we obtain the radiation power at the frequency $2 \omega$ in the linear regime

$$
\mathcal{P}_{\operatorname{rad}}(2 \omega)=\approx \frac{\varepsilon_{c} \Phi_{0}^{2} \omega_{p}^{4}}{2 \pi^{3} c s^{2} \ell^{2} \omega^{2} b^{4}} \frac{N}{k_{\omega} s} \frac{\left[\cos ^{2}(2 \tilde{\omega} \tilde{l})-2 \cos (2 \tilde{\omega} \tilde{l}) \cos (b \tilde{l}+1][1-\cos (\tilde{b})]\right.}{\sin ^{2}(2 \tilde{\omega} \tilde{l})+\left(\alpha_{\mathrm{tr}} \tilde{l}\right)^{2} \cos ^{2}(2 \tilde{\omega} \tilde{l})}
$$


Here, as for rectangular lattice, we have large factor $\left(k_{\omega} s\right)^{-1}$ due to super-radiation at the frequency $2 \omega$. This differs from the radiation power for rectangular lattice at the same frequency by the factor of order unity at $\tilde{\omega} \sim 1$ and $b \sim 1$ in the linear regime. Thus both rectangular and triangular lattice give approximately the same radiation power for $\tilde{\omega} \sim 1$ and in both cases the radiation is coherent. However, their $I-V$ characteristics and the conversion coefficients $Q$ are very different. The fraction of the power converted into radiation depends on quasiparticle conductivities $\sigma_{a b}$ and $\sigma_{c}$. These conductivities remain nonzero in cuprate superconductors even if temperature approaches zero because cuprates are gapless superconductors. As a result, only small part, of order $\left(b^{2} \ell^{2} \alpha_{\mathrm{tr}} \tilde{l}\right)^{-1}$, is converted into the radiation in the linear regime. Thus for triangular lattice we estimate very small $Q \propto \ell^{-2}$. This translates into strong heating and practically triangular lattice cannot provide a realistic source of radiation.

The $I-V$ characteristics is determined by the behavior of $\phi$ and so it is resonant at low dissipation, as in a single JJ. In particular, for (i) weak dissipation, $q \approx 2 \tilde{\omega}+i \alpha_{\mathrm{tr}}$, and (ii) small frequencies $\tilde{\omega} \ll b / 2$ we obtain

$$
i_{J} \approx \frac{8}{b^{4}} \alpha_{b} \tilde{\omega}+\frac{2 \alpha_{\mathrm{b}}[\cos (2 \tilde{\omega} \tilde{l})-\cos (b \tilde{l})] \cos (2 \tilde{\omega} \tilde{l})}{\tilde{l} \tilde{\omega} b^{2}\left[\sin ^{2}(2 \tilde{\omega} \tilde{l})+\left(\alpha_{\mathrm{tr}} l\right)^{2} \cos ^{2}(2 \tilde{\omega} \tilde{l})\right]}
$$

where $\alpha_{b}=\nu_{c}+\nu_{a b} b^{2}$ and $\alpha_{\mathrm{tr}}=\nu_{c}+\nu_{a b} \tilde{\omega}^{2}$. The amplitude of the Fiske peak at $2 \tilde{\omega}_{n} \tilde{l}=\pi n$ is

$$
\delta_{n} i_{J} \approx \frac{2\left[1-(-1)^{n} \cos (b \tilde{l})\right]}{\tilde{\omega}_{n} b^{2} \tilde{l}^{3}\left(\nu_{c}+\nu_{a b} \tilde{\omega}_{n}^{2}\right)} .
$$

Note that triangular lattice excites resonances for the antiphase modes whose frequencies are by factor $2 \ell=2 \lambda \mathrm{ab} / \mathrm{s} \sim 300$ smaller then the frequencies of the homogeneous modes excited by the rectangular lattice. Very pronounced Fiske steps have been observed recently by Kim et al. [23]

We also estimated that the lattice with random parameters $\kappa_{n}$ gives incoherent radiation with approximately the same power and the conversion coefficient as triangular lattice.

\section{Conclusions}

We have estimated the radiation power from most probable vortex lattices in the large- $N$ limit. Our estimate for rectangular lattice in BSCCO agrees satisfactory with results of numerical study by Tachiki et al. [27]. We see that only rectangular lattice gives significant radiation power and quite high conversion coefficient, i.e. up to half power fed into the crystal may be converted into 
the radiation. Thus, not very high currents are needed to get significant radiation power. However, the main question to be answered is what are parameters of the stability of rectangular lattice in finite-size samples. Other open questions include:

1. What is the radiation power in highly nonlinear regime for triangular lattice and for rectangular lattice with moderate number of layers $N$ ?

2. What is the structure of steady states and the radiation power of crystals with not very large $N<\ell$, when the boundaries along the $y$-axis become important?

3. What are boundary conditions and the radiation power in crystals with $w k_{\omega} \ll 1$ ?

4. Are there fully gapped layered superconducting materials or artificial multilayer system which can be used as radiation sources? Presence of finite gap in the whole Fermi surface drastically reduces quasiparticle dissipation at low temperatures and the problem of high dissipation for the moving triangular lattice in the high-temperature superconductors will be avoided.

5. Is it possible to get stronger radiation from the triangular lattice by modulating the radiated edge of the crystal? For example, one can use some periodic layer of dielectric or metal between the crystal and the air which may result in more effective conversion of high $k_{y}$ electromagnetic fields inside the crystal into low $k_{y}$ outside electromagnetic waves. For that the surface modulation has to have the periodicity $2 s$, i.e., every second layer has to be closed. For efficient conversion, one can anticipate that the radiation power would be larger by the factor $\ell^{2} \approx 10^{4}$ in BSCCO and TBCCO crystals.

The authors thank Yu. Galperin, A. Gurevich, I. Martin, K. Rasmussen, N. Grønbech-Jensen, and R. Kleiner for useful discussions. This research was supported by the Department of Energy under contracts \# W-7405-ENG-36 (LANL) and \# W-31-109-ENG-38 (Argonne).

[1] B.D. Josephson, Phys. Lett. 1, 251 (1962).

[2] I.M. Dmitrenko, I.K. Yanson, and V.M. Svistunov, Pis'ma ZhETF, 2, 134 (1965); I.K. Yanson, Low Temp. Phys. 30, 516 (2004).

[3] D.N. Langenberg, D.J Scalapino, B.N. Taylor, and R.E. Rice, Phys. Rev. Lett., 15, 294 (1965). 
[4] J. Swihart, J. Appl. Phys. 32, 461 (1961).

[5] I.O. Kulik and I.K. Yanson, The Josephson Effect in Superconducting Tunneling Structures, Jerusalem, 1972.

[6] A.V. Ustinov, Physica D, 123, 315 (1998).

[7] N.F. Pedersen and A.V. Ustinov, Superc. Sci. Technol. 8, 389 (1995).

[8] A.K. Jain, K.K. Likharev, J.E. Lukens, and J.E Sauvageau, Phys. Rep. 109, 309 (1984).

[9] R. Kleiner, Phys. Rev. B, 50, 6919 (1994).

[10] Ustinov and S. Sakai, Appl. Phys. Lett., 73, 686 (1998).

[11] Yu. M. Ivanchenko, Phys. Rev. B, 54, 13247 (1996).

[12] M. Darula, T. Doderer, and S. Beuven, Supercond. Sci. Technol. 12, R1 (1999).

[13] W.E. Lawrence and S. Doniach, in Proceedings of the 12th International Conference on Low Temperature Physics, Kyoto 1970, edited by E. Kanda (Keigaku, Tokyo, 1970).

[14] R. Kleiner, F. Steinmeyer, and P. Müller, Phys. Rev. Lett. 68, 2394 (1992); R. Kleiner and P. Müller, Phys. Rev. B 49, 1327 (1994).

[15] Yu. Latyshev, J.E. Nevelskaya, P. Monceau, Phizika Nizkikh Temperatur, 22, 629 (1996).

[16] M.B. Gaifullin, Y. Matsuda, and L.N. Bulaevskii, Phys. Rev. Lett. 81, 3551 (1998).

[17] O.K.C. Tsui, N.P. Ong, Y. Matsuda, Y.F. Yan, and J.B. Peterson, Phys. Rev. Lett. 73, 724 (1994); O.K.C. Tsui, N.P. Ong, and J.B. Peterson, Phys. Rev. Lett. 76, 819 (1996).

[18] Y. Matsuda, M.B. Gaifullin, K. Kumagai, K. Kadowaki, and T. Mochiku, Phys. Rev. Lett. 75, 4512 (1995); Y. Matsuda, M.B. Gaifullin, K. Kumagai, K. Kadowaki, and K. Hirata, Phys. Rev. Lett. 78, 1972 (1997).

[19] H. B. Wang, P. H. Wu, and T. Yamashita, Phys. Rev. Lett. 87, 107002 (2001).

[20] Yu.I. Latyshev, M.B. Gaifullin, T. Yamashita, M. Machida, and Y. Matsuda, Phys. Rev. Lett. 87, 247007 (2001).

[21] A. Irie, Y. Hirai, and G. Oya, Appl. Phys. Lett. 72, 2159 (1998).

[22] V. M. Krasnov, N. Mros, A. Yurgens, and D. Winkler, Phys. Rev. B 59, 8463 (1999).

[23] S.-M. Kim, H. B. Wang, T. Hatano, S. Urayama, S. Kawakami, M. Nagao, T. Takano, T. Yamashita, and K. Lee, Phys. Rev. B 72, R140504 (2005).

[24] T. Koyama and M. Tachiki, Sol. St. Comm. 96, 367 (1995).

[25] Yu.I. Latyshev, M.B. Gaifullin, T. Yamashita, M. Machida, and Y.Matsuda, Phys. Rev. Lett., 87, 247007 (2001). 
[26] S.N. Artemenko and S.V. Remizov, Physica C, 362, 200 (2000).

[27] M. Tachiki, M. Iizuka, S. Tejima, and H. Nakamura, Phys. Rev. B, 71, 134515 (2005).

[28] S.Savel'ev, V. Yampol'skii, F. Nori, Phys. Rev. Lett. 95, 187002 (2005); S.Savel'ev, A.L. Rachmanov, F. Nori, Phys. Rev. Lett. 94, 157004 (2005); Phys. Rev. B, 72, 144515 (2005).

[29] G. Hechtfischer, R. Kleiner, A.V. Ustinov, and P. Müller, Phys. Rev. Lett. 79,1365 (1997).

[30] K. Kadowaki, Joint JSPS and ESF Conference on Vortex Matter in Nanostructured Superconductors (VORTEX IV) 3-9 September 2005, Crete, Greece.

[31] I.E. Batov, X.Y. Jin, S.V. Shitov, V. Koval, P. Müller, and A.V. Ustinov, preprint.

[32] I.O. Kulik, Pis'ma ZhETF, 2, 134 (1965) [JETP Lett, 2, 84 (1965)].

[33] M. Cirillo, N. Grønbech-Jensen, M.R. Samuelsen, M. Salerno, and G. V. Rinati, Phys. Rev. B, 58, 12377 (1998).

[34] J.-J. Chang. Phys. Rev. B, 28, 1276 (1983).

[35] R. Simmonds, K.M. Lang, D.A. Hite, D.P. Pappas, and J.M. Martinis, Phys. Rev. Lett., 93, 035301 (2004).

[36] A. Gurevich, Phys. Rev. B 46, 3187 (1992), 48, 12857 (1993).

[37] L.N. Bulaevskii, M. Zamora, D. Baeriswyl, H. Beck, and J.R. Clem, Phys. Rev. B 50, 12831 (1994).

[38] R. Kleiner, P. Müller, H. Kohlstedt, N.F. Pedersen, and S. Sakai, Phys. Rev. B 50, 3942 (1994).

[39] L.N. Bulaevskii, D. Dominguez, M.P. Maley, A.R. Bishop, and B.I. Ivlev, Phys. Rev. B, 53, 14601, (1996)

[40] A.E. Koshelev and L.N. Bulaevskii, Phys. Rev. B, 62, 14403 (2000).

[41] A.E. Koshelev and I. Aranson, Phys. Rev. Lett. 85, 3938 (2000); Phys. Rev. B 64, 174508 (2001).

[42] S.N. Artemenko and S.V. Remizov, Phys. Rev. B, 67, 144516 (2003).

[43] A. E. Koshelev, Physica C, 437-438, 157,(2006).

[44] S. Ooi, T. Mochiku, and K. Hirata, Phys. Rev. Lett. 89, 247002 (2002).

[45] Yu.I. Latyshev, A.E. Koshelev and Bulaevskii, Phys. Rev. B, 68, 134504 (2003).

[46] V.K. Thorsmolle, R.D. Averitt, M.P. Maley, L.N. Bulaevskii, C. Helm, and A.J. Taylor, Opt. Lett. 26, $1292(2001)$. 